\title{
Brownian Dynamics Simulations with Hard-Body Interactions: Spherical Particles
}

\author{
Hans Behringer, a) and Ralf Eichhorn', b) \\ 1) Johannes Gutenberg-Universität Mainz, Institut für Physik, Staudinger Weg 7, D-55128 Mainz, \\ Germany \\ ${ }^{2)}$ Nordic Institute for Theoretical Physics (NORDITA), Royal Institute of Technology and Stockholm University, \\ Roslagstullsbacken 23, 10691 Stockholm, Sweden
}

A novel approach to account for hard-body interactions in (overdamped) Brownian dynamics simulations is proposed for systems with non-vanishing force fields. The scheme exploits the analytically known transition probability for a Brownian particle on a one-dimensional half-line. The motion of a Brownian particle is decomposed into a component that is affected by hard-body interactions and into components that are unaffected. The hard-body interactions are incorporated by replacing the 'affected' component of motion by the evolution on a half-line. It is discussed under which circumstances this approach is justified. In particular, the algorithm is developed and formulated for systems with space-fixed obstacles and for systems comprising spherical particles. The validity and justification of the algorithm is investigated numerically by looking at exemplary model systems of soft matter, namely at colloids in flow fields and at protein interactions. Furthermore, a thorough discussion of properties of other heuristic algorithms is carried out.

\section{INTRODUCTION}

Many physical processes in soft matter systems, like colloids $s^{1-3}$, polymers $\underline{4}^{\underline{4}}$ or biomolecular systems ${ }^{\underline{5}}$, can be modeled in terms of Langevin equations where the influence of the solvent is captured by friction and random forces $\underline{\underline{6}-9}$ The separation of the respective length and time scales of the solvent particles and the constituent particles of soft matter typically allows one to use the high friction limit where the velocities of the latter particles are strongly damped and inertia effects are thus negligible (overdamped Langevin equations). Another common idealization in the modeling of mesoscopic physical systems within the framework of Langevin equations consists in representing the extremely shortranged and strong repulsive contact forces between particles or between particles and space-fixed obstacles or walls by hard-body interactions. Important examples for physical processes which are investigated using these limits include the dynamical behavior of polymers and colloids in dilute as well as concentrated solutions, the self-assembly of biomolecules into biological structures like membranes or protein aggregates, the motion of molecules in biomolecular systems and the migration of macromolecules in microfluidic channels $\underline{10}$.

To unravel the properties of such physical processes, a thorough investigation of the particles' trajectories is often indispensable. To name just two examples, the knowledge of the typical trajectories is crucial to analyze association pathways of complex-forming proteins or the migration mechanisms of biomolecules in microfluidic channels. As in most cases an analytical treatment of the Langevin equations of motion is not possible on such detailed, trajectory-wise level, efficient numerical inte-

\footnotetext{
a) Electronic mail: behringh@uni-mainz.de

b) Electronic mail: eichhorn@nordita.org
}

gration schemes for solving these stochastic differential equations are essential ${ }_{11,12}^{12}$ These schemes require a certain degree of regularity of the involved force fields, 11 so that hard-body interactions (associated with reflective boundaries 13 ) can not be integrated directly due to their singular nature. Therefore, it is necessary to represent the effect of singular hard-body interactions in a tractable form. In order to avoid the numerically costly introduction of steep (and regular) auxiliary potentials, alternative potential-free methods seem particularly promising with respect to computational efficiency. Such methods are based on detecting 'unphysical configurations', i e. 'collisions' or 'overlaps' between hard-body entities that arise from a regular numerical integration step, and on some rule that specifies how this unphysical configuration is to be corrected in order to create a physically valid configuration. Several heuristic methods along these lines have been discussed in the literature, ranging from Monte Carlo type schemes, where unphysical configurations are just rejected, to methods where overlapping particles with hard cores are displaced along their connection line. This is done by applying specific rules that are based on geometric considerations or on the physics of elastic collisions $\underline{\underline{14}} \underline{-16}$ In recent years the emergence of event-driven molecular dynamics has inspired the development of trajectory-based heuristic 'event-driven' Brownian dynamics schemes 17,18 These methods often lack a thorough justification. $\underline{19}$ Apart from the rejection method 14 and the event-driven scheme of Tao et al $\underline{\underline{18}}$ it is not obvious how the different methods can be applied to driven diffusive systems with additional non-vanishing force fields.

In this work we propose a novel method to account for hard-body interactions in numerical Brownian dynamics simulations. The new approach reverts to the transition probability for the time evolution of a particle whenever a hard-body interaction has to be accounted for. This transition probability is determined by the Fokker-Planck 
equation which provides an alternative way to describe the physics of stochastic processes ${ }^{6}-\underline{\underline{8}}$ Once this transition probability is known, a physical trajectory of a particle can be directly generated. However, for many systems this transition probability is not known analytically. In this paper we will argue and discuss how the analytical solution of the Fokker Planck equation for the one-dimensional half-line can be used to account for hardbody interactions in general, more-dimensional systems. The new algorithm has the advantage that it can be applied to systems with spatially varying force-fields and can be used independently of the applied numerical integration scheme. In addition, the effects of the introduced approximations due to applying the transition probability for a system on a half-line can be critically assessed so that these approximations can be justified and numerically controlled.

The article is organized as follows. In Sec. II the basics of Brownian dynamics simulations are recapitulated. Then the problem of accounting for hard-body interactions in Brownian dynamics is discussed and the basic idea of our novel approach to incorporate them is outlined. In this context other proposed heuristic methods are discussed in Sec. III Section IV is then devoted to the development of the new algorithm for space-fixed hard walls. The algorithm is next generalized to systems with hard spheres in Sec. D The validity, quality and efficiency of the methods are scrutinized in Sec. VI by numerically investigating different exemplary physical situations with non-vanishing force fields. A brief account of some of the results of this article was already published elsewhere $\stackrel{20}{\underline{20}}$

\section{HARD-BODY INTERACTIONS IN BROWNIAN DYNAMICS}

\section{A. Basics of Brownian Dynamics simulations}

Consider a Brownian particle in a solution and in a force field $\vec{F}=\vec{F}(\vec{r}, t)$ at temperature $T$. Apart from the deterministic force $\vec{F}$ due to external potentials, non-conservative forces or inter-particle interactions, the Brownian particle experiences the influence of the medium. One influence is related to the friction which is determined by Stokes' friction force $-\eta \dot{\vec{r}}$ with friction coefficient $\eta$ and particle velocity $\dot{\vec{r}}$. In addition, the medium influences the particle by random collisions through its constituents. This thermal heat bath is represented by a random force $\vec{\xi}$ with the statistical properties of Gaussian random variables: $\left\langle\xi^{\alpha}(t) \xi^{\beta}(s)\right\rangle=\delta^{\alpha \beta} \delta(t-s)$ with $\alpha$ and $\beta$ denoting Cartesian components. In the strong friction limit, for which inertia effects are negligible, the evolution of the particle is then governed by the Langevin equation

$$
\dot{\vec{r}}(t)=\frac{\vec{F}(\vec{r}(t), t)}{\eta}+\sqrt{2 D} \vec{\xi}(t),
$$

where the strength of the fluctuating force is related to the diffusion constant $D=k_{\mathrm{B}} T / \eta$ (overdamped Langevin dynamics, Brownian dynamics) $\frac{3,7.12}{1}$ Note that the assumption of the strong friction limit is well satisfied for biological, colloidal or polymeric systems $\underline{4.5}$

The Langevin evolution equation can be used to obtain the trajectory of the particle whose physical contents can then be investigated. In this context the Langevin equation is best formulated in terms of differentials,

$$
\mathrm{d} \vec{r}=\vec{v} \mathrm{~d} t+\sqrt{2 D} \mathrm{~d} \vec{W} .
$$

Here the drift term is represented in terms of a velocity $\vec{v}=\vec{F} / \eta$ and the stochastic term is related to a Wiener process $\vec{W}$. The Wiener process $\vec{W}$ describes free Brownian diffusion and is mathematically defined to be a Gaussian stochastic process with mean $\left\langle W^{\alpha}(t)\right\rangle=0$ and covariance $\left\langle W^{\alpha}(t) W^{\beta}(s)\right\rangle=\delta^{\alpha \beta} \min (t, s) \underline{\underline{6,21,22}}$ In addition, the Itô Lemma $\left\langle\mathrm{d} W^{\alpha}(t) \mathrm{d} W^{\beta}(t)\right\rangle=\mathrm{d} t \delta^{\alpha \beta}$ holds true for its differential. Note that the incremental Wiener process $\mathrm{d} \vec{W}(t)$ cannot be written in the form $\vec{w}(t) \mathrm{d} t$ due to the non-differentiability of the trajectory of a freely diffusing particle $\underline{\underline{6}, 21,22}$

The numerical integration of (2) will generate a discrete approximation of the trajectory. To this end a discrete 'incremental' displacement corresponding to (2) is generated for a finite time step from the current physical position of the particle. The most prominent integration scheme for such Brownian dynamics simulations is the Euler algorithm (see Sec. III). Apart from single step algorithms like the Euler method, multistep integration schemes such as the Heun algorithm have been developed that additionally incorporate the information from configurations for intermediate steps (more details about the numerical integration of stochastic differential equations, in particular on necessary conditions on the regularity of the involved forces, can be found in the literature $3,11,12,23)$.

One crucial point in carrying out a numerical integration of (2) is the choice of an appropriate size of the time step between two successive times of the discrete trajectory. For a non-zero and spatially varying force field $\vec{F}$, the time step has to be small enough so that the force is virtually constant on the scale that is associated with the typically proposed spatial displacement. For systems whose time evolution is usually modelled within a Brownian dynamics approach, however, the corresponding force fields are in general relatively smooth. This allows the use of fairly large integration time steps.

\section{B. Algorithm for accounting hard-body interactions}

For systems with hard-body interactions the direct numerical integration of the unmodified Langevin equations over a given time step can result in unphysical configurations where the particles are displaced into the wall, for example, or where hard-core particles penetrate each 
other. Such configurations, which are obtained from the direct integration of the Langevin equation but are not consistent with hard-body interactions will be called 'unphysical' in the following. As the hard-body interactions are singular in nature they cannot directly be incorporated into the integration scheme. One possibility is given by a representation in terms of regular potentials. This approach, however, has the drawback, that the auxiliary potentials have to be rather steep and therefore the acceptable time step for the numerical integration has to be very small rendering the simulation extremely timeconsuming. Thus other more effective potential-free ways to account for hard-body interactions are desirable $\underline{\underline{24}}$

The method we propose here is based on the approach to consider the physics of a diffusing particle in a force field $\vec{F}$ by directly looking at the transition probability $p\left(\vec{r}, t ; \vec{r}_{0}, t_{0}\right)$ to find the particle at position $\vec{r}$ at time $t$ provided it was at position $\vec{r}_{0}$ at time $t_{0}<t$. This (normalized) probability is governed by the Fokker-Planck equation $\underline{\underline{6}-\underline{\underline{8}}}$

$$
\frac{\partial}{\partial t} p\left(\vec{r}, t ; \vec{r}_{0}, t_{0}\right)=\left(D \Delta_{\vec{r}}-\vec{\nabla}_{\vec{r}} \cdot \frac{\vec{F}(\vec{r}, t)}{\eta}\right) p\left(\vec{r}, t ; \vec{r}_{0}, t_{0}\right) .
$$

To solve this partial differential equation boundary conditions for the physical domain have to be provided. The presence of hard walls at a boundary with normal vector $\vec{n}$ corresponds to reflective boundary conditions for which the normal projection $\vec{n} \cdot \vec{j}$ of the probability flux

$$
\vec{j}\left(\vec{r}, t ; \vec{r}_{0}, t_{0}\right)=-\left(D \vec{\nabla}_{\vec{r}}-\frac{\vec{F}(\vec{r}, t)}{\eta}\right) p\left(\vec{r}, t ; \vec{r}_{0}, t_{0}\right)
$$

vanishes at the boundary.

In general, a realization for the displacement $\vec{r}-\vec{r}_{0}$ of a Brownian particle after elapsed time $t-t_{0}$ can be directly generated from the transition probability $p\left(\vec{r}, t ; \vec{r}_{0}, t_{0}\right)$. Suppose now that this probability is known in the vicinity of the hard surface and that the numerical integration using the unmodified Langevin equation (without hard walls) has led to an unphysical position $\vec{r}$ for the particle that was initially at $\vec{r}_{0}$. Instead of applying a modified integration step to the Langevin equation, a new physical position $\vec{r}^{*}$ for the elapsed time $t$ is directly generated from that part of the transition probability $p\left(\vec{r}^{*}, t ; \vec{r}_{0}, 0\right)$ that represents the particle's encounter with the wall. This recourse to the transition probability whenever a hard-body interaction has to be accounted for forms the heart of our proposed algorithm. In the following we will demonstrate that the transition probability $p\left(\vec{r}, t ; \vec{r}_{0}, t_{0}\right)$ for spherical particles can indeed be decomposed in a mathematically rigorous way into a 'free' part (without hard-body interactions) and a part that stems from the reflective hard boundary. This decomposition is based on the solution of the Fokker-Planck equation on a onedimensional half-line with a reflecting boundary at the origin.

\section{Exact one-dimensional algorithm}

For the particular example of a one-dimensional system, where the position $q$ is restricted to the half-line, $[0, \infty[$ and is subject to the constant force $f$ the FokkerPlanck equation reads

$$
\frac{\partial}{\partial t} p=D_{q} \frac{\partial^{2}}{\partial q^{2}} p-\frac{f}{\eta} \frac{\partial}{\partial q} p .
$$

The transition probability can be calculated analytically for a reflective boundary at $q=0,25,26$ Using the notation $v:=f / \eta$ and setting $t_{0}=0$ without loss of generality the solution for the transition probability $p\left(q, t ; q_{0}\right) \equiv$ $p\left(q, t ; q_{0}, t_{0}=0\right)$ is given by

$$
p\left(q, t ; q_{0}\right)=p_{1}\left(q, t ; q_{0}\right)+p_{2}\left(q, t ; q_{0}\right)+p_{3}\left(q, t ; q_{0}\right)
$$

with the contributions

$$
\begin{aligned}
& p_{1}\left(q, t ; q_{0}\right)=\frac{1}{\sqrt{4 \pi D_{q} t}} \exp \left(-\frac{\left(q-q_{0}-v t\right)^{2}}{4 D_{q} t}\right), \\
& p_{2}\left(q, t ; q_{0}\right)=\frac{\exp \left(-\frac{v q_{0}}{D_{q}}\right)}{\sqrt{4 \pi D_{q} t}} \exp \left(-\frac{\left(q+q_{0}-v t\right)^{2}}{4 D_{q} t}\right)
\end{aligned}
$$

and

$$
p_{3}\left(q, t ; q_{0}\right)=-\frac{v}{2 D_{q}} \exp \left(\frac{v q}{D_{q}}\right) \operatorname{erfc}\left(\frac{q+q_{0}+v t}{\sqrt{4 D_{q} t}}\right)(.7 \mathrm{c})
$$

Each $p_{i}$ satisfies the Fokker-Planck equation (5) individually, their sum then satisfies the initial condition $p\left(q, t=0 ; q_{0}\right)=\delta\left(q-q_{0}\right)$, the reflective boundary condition $\left.j\left(q, t ; q_{0}\right)\right|_{q=0}=-\left.\left(D_{q} \frac{\partial}{\partial x} p-v p\right)\right|_{q=0}=0$, and is normalized on the half-line $[0, \infty[$. The different parts have the following intuitive interpretation. The first term $p_{1}$ is the probability distribution that corresponds to unrestricted diffusion in a constant force field and represents the contributions from particles that do not encounter the wall at $q=0$ during the propagation over the time $t$. The propagation of such particles is already correctly captured by the 'free' Langevin equation $\mathrm{d} q=v \mathrm{~d} t+\sqrt{2 D_{q}} \mathrm{~d} W$ corresponding to (5) without boundary at $q=0$. The second and third terms contain the contributions from particles that encounter the wall during the propagation time $t$. These contributions $p_{2}$ and $p_{3}$ are obviously not correctly contained in the associated Langevin equation without further consideration of the reflective boundary condition at $q=0$.

\section{The algorithm}

It is now obvious how physical positions that are consistent with a reflective boundary at $q=0$ can be generated:

1. Starting at $q_{0}>0$, suggest a new position $q$ of the Brownian particle (with diffusion constant $D_{q}$ ) 
after time step $\mathrm{d} t$ by some given numerical integration scheme for the Langevin equation (without further consideration of the hard wall).

2 a. If the proposed $q$ is in the physical domain, i.e. $q>0$, it is accepted as the new particle position.

$2 \mathrm{~b}$. If the proposed position is unphysical, i.e. $q<$ 0 , a new position is generated directly from the transition probability (see Appendix A)

$$
p_{\mathrm{H}}\left(q, q_{0}, \mathrm{~d} t, v, D_{q}\right)=\frac{p_{2}(q)+p_{3}(q)}{\int_{0}^{\infty} \mathrm{d} q\left(p_{2}(q)+p_{3}(q)\right)}
$$

Obviously, the end positions which are directly accepted from the integration step of the Langevin equation make up the contribution $p_{1}$ in the exact solution (6) of the transition probability. The distribution $p_{\mathrm{H}}$ represents the (normalized) part $p_{2}+p_{3}$ in the analytical solution (6) capturing the physics of particles that encounter the wall at least once. See Appendix $\mathrm{A}$ on how to generate $q$ from (8) in practice.

\section{Discussion}

The algorithm is constructed such that a stochastic particle trajectory is generated from two contributions: Parts where the particle does not encounter the wall at all are captured by a standard integration step, whereas the parts of the trajectory where wall-collisions occur are constructed from $p_{\mathrm{H}}$. Let us look at this point from a different angle. Mathematically speaking the algorithm uses two random variables $X_{\mathrm{G}}$ and $X_{\mathrm{H}}$ to construct a valid end position $X$. The Gaussian variable $X_{\mathrm{G}}$ describes unrestricted free diffusion, can thus take on all real numbers $\mathbb{R}$ and is distributed according to the Gaussian distribution $p_{1}$ (compare (7a) ), which is normalized on $\mathbb{R} . X_{\mathrm{H}}$ can take on only positive real numbers $\mathbb{R}_{>0}$ and is distributed according to (the normalized) $p_{\mathrm{H}}$ of relation (8). The final random variable generated from the algorithm is of the form

$$
X=X_{\mathrm{G}} \theta\left(X_{\mathrm{G}}\right)+X_{\mathrm{H}} \theta\left(-X_{\mathrm{G}}\right)
$$

where $\theta(X)$ is the Heaviside step function. Defining the collision probability

$$
\begin{aligned}
w & =\int_{0}^{\infty} \mathrm{d} q\left(p_{2}(q)+p_{3}(q)\right) \\
& =\int_{0}^{\infty} \mathrm{d} q\left(1-p_{1}(q)\right)=\int_{-\infty}^{0} \mathrm{~d} q p_{1}(q) \\
& =\frac{1}{2} \operatorname{erfc}\left(\frac{q_{0}+v \mathrm{~d} t}{\sqrt{4 D_{q} \mathrm{~d} t}}\right)
\end{aligned}
$$

one can write $p_{\mathrm{H}}=\left(p_{2}+p_{3}\right) / w$. The random variable $X$ associated with the algorithm has the distribution function

$$
\begin{aligned}
p_{\mathrm{alg}}(q)= & \int_{-\infty}^{+\infty} \mathrm{d} x_{1} \int_{0}^{\infty} \mathrm{d} x_{2} p_{\mathrm{G}}\left(x_{1}\right) p_{\mathrm{H}}\left(x_{2}\right) \\
& \times \delta\left(q-\left[x_{1} \theta\left(x_{1}\right)+x_{2} \theta\left(-x_{1}\right)\right]\right) \\
= & \int_{-\infty}^{+\infty} \mathrm{d} x_{1} \int_{0}^{\infty} \mathrm{d} x_{2} p_{1}\left(x_{1}\right) \frac{p_{2}\left(x_{2}\right)+p_{3}\left(x_{2}\right)}{w} \\
& \times \delta\left(q-\left[x_{1} \theta\left(x_{1}\right)+x_{2} \theta\left(-x_{1}\right)\right]\right) .
\end{aligned}
$$

Due to the Heaviside functions in the argument of the delta function the latter can be rewritten as $\delta(q-$ $\left.\left[x_{1} \theta\left(x_{1}\right)+x_{2} \theta\left(-x_{1}\right)\right]\right)=\theta\left(x_{1}\right) \delta\left(q-x_{1}\right)+\theta\left(-x_{1}\right) \delta\left(q-x_{2}\right)$. The resulting two integrals finally add up to $p_{\text {alg }}(q)=$ $p_{1}(q)+p_{2}(q)+p_{3}(q)$ and hence the algorithm indeed generates random variables which are distributed according to the Smolouchowski solution (6).

By construction our proposed procedure for a simulation in a system with constant force will generate a trajectory with the correct statistics independent of the size of the used time step $\mathrm{d} t$. For a slowly varying force the time step can be chosen small enough so that the force stays virtually constant for the typical displacement of the particle. For each time step the simulation is then carried out as above with the presently acting force (see Appendix B for an illustration). For such an appropriately adjusted time step the Brownian dynamics simulation will generate a trajectory that exhibits the correct physical properties in a controllable accuracy.

For the outlined approach to work for a general physical system one has to solve the Fokker-Planck equation for the transition probability in the vicinity of a hard surface analytically. This is only possible in closed form for a very limited number of problems. In the following sections we will show in details that the solution for the one-dimensional half-line can be used to construct an approximate transition probability with which hardbody interactions can be accounted for in many situations. The approximation is thereby controlled by the size of the time step. For small time steps only small displacements show up so that a generally shaped hard surface appears to be virtually flat. For the normal direction the systems is then basically restricted to a half-line whereas the motion in the components parallel to the surface is unrestricted. This parallel motion is decoupled from the normal motion and can thus be treated within the usual approach whereas the one-dimensional normal motion is dealt with by the one-dimensional approach from above. Details will be worked out below. We note already at this point, however, that the involved adaption of the time step for spatially structured hard boundaries works along the same lines as the corresponding choice to capture the effect of spatially varying force fields. 


\section{HEURISTIC REPRESENTATIONS OF HARD WALLS}

Before extending the outlined approach to more complex physical systems we discuss the quality and validity of other proposed heuristic methods to account for hard-body interactions in a potential-free manner. To this end we consider a Brownian particle on the onedimensional half-line in a constant force field for which the analytical solution (6) is at hand. This special system will enable us to precisely identify the shortcomings of those schemes. In the following we will only compare methods that can be directly extended to systems with non-vanishing forces.

\section{A. Rejection scheme}

A first possible scheme could consist in discarding all displacements into hard walls as unphysical and repeating the integration step until a valid displacement is obtained. However, in doing so the influence of the contributions $p_{2}$ and $p_{3}$ is not captured even approximately, as this scheme tries to reconstructed the whole transition probability only from (the renormalized) $p_{1}$. This results in a very poor convergence in the limit of decreasing integration time step $\mathrm{d} t$ as can be seen in Fig. 11 (left panel). In particular, one would expect that systematic deviations show up only in close proximity of the wall. The mentioned renormalizing of $p_{1}$, however, leads to deviations even far away from the wall (compare Fig. 1, left panel).

The Monte Carlo inspired rejection scheme models the effect of hard body interactions by dismissing a proposed propagation that leads to an unphysical configuration, but still advancing time. ${ }^{14}$ The part $p_{1}$ in the probability distribution (6) is correctly reproduced for a time step $\mathrm{d} t$, but the contributions from $p_{2}$ and $p_{3}$ are replaced by a delta function situated at the initial position $q_{0}$ with a weight given by the collision probability $w$ (compare 10), see also Fig. 1 (right panel) and Fig. 21). The one-step distribution function of the rejection scheme thus reads

$$
p_{\mathrm{re}}(q)=p_{1}(q)+\frac{1}{2} \operatorname{erfc}\left(\frac{q_{0}+v \mathrm{~d} t}{\sqrt{4 D_{q} \mathrm{~d} t}}\right) \delta\left(q-q_{0}\right) .
$$

In this way the relative weight of the contributions $p_{1}$ and $p_{2}+p_{3}$ is correctly captured, although the influence of encounter events is not 'explicitly' worked in. In addition, the reflective boundary condition at $q=0$ is violated. Instead of a vanishing probability flux one has

$$
\left.j_{\mathrm{re}}\right|_{q=0}=-\frac{\exp \left(-\frac{\left(q_{0}+v \mathrm{~d} t\right)^{2}}{4 D_{q} \mathrm{~d} t}\right)}{\sqrt{4 \pi D_{q} \mathrm{~d} t}} \frac{q_{0}-v \mathrm{~d} t}{2 \mathrm{~d} t} .
$$

In practise a finite time step will give acceptable results for large initial separations from the wall where the typical displacement will not lead to an encounter. Close to the wall where $\sqrt{2 D_{q} \mathrm{~d} t} / q_{0}$ is considerably larger than zero, however, systematic deviations show up. For illustration we give (the asymptotic series of) the mean value $\langle q\rangle_{\text {re }}$ of (16)

$$
\langle q\rangle_{\mathrm{re}}=\langle q\rangle-\frac{\mathrm{e}^{-\frac{q_{0}^{2}}{4 D_{q} \mathrm{~d} t}}}{\sqrt{4 \pi D_{q} \mathrm{~d} t}}\left(\mathrm{e}^{-\frac{v q_{0}}{2 D_{q}}} 2 D_{q} \mathrm{~d} t+\mathcal{O}\left(\mathrm{d} t^{2}\right)\right)
$$

where $\langle q\rangle$ is the mean of the exact solution (6]). (In Refs. 14 and 17 the convergence of the rejection method in the limit of vanishing $\mathrm{d} t$ was discussed for systems without deterministic forces). Figure 1 (right panel) illustrates the influence of the size of the time step on the convergence behavior. The chosen parameters correspond to a situation where the probability to encounter the wall is already moderately small. Even then rather small time steps have to be used to reduce the systematic deviations for end positions close to the wall.

We note that a modification of the rejection scheme was proposed in Ref. 27 for many-particle systems. There the amount for which time is advanced during one step is related to the fraction of proposed displacements without unphysical hard-core overlaps compared to displacements with overlaps (rejection with 'intrinsic' clock). For just one particle, this scheme corresponds to the rejection method without advancing time, which has been demonstrated above (see also Fig. 1, left panel) to exhibit poor convergence.

\section{B. Event-driven methods}

The event-driven scheme of Tao et al. $\frac{18}{18}$ attempts to capture the influence of encounter events by using an auxiliary path for the propagating particle. For an integration step where an overlap is detected the singular trajectory of a Brownian particle is replaced by a regular path whose parameterization is obtained from the used integration scheme. To keep the discussion clear we consider the Euler algorithm where the new particle position $q(t+\mathrm{d} t)$ is given by

$$
q(t+\mathrm{d} t)=q(t)+v(t) \mathrm{d} t+\sqrt{2 D_{q} \mathrm{~d} t} G
$$

with $G$ being a random Gaussian variable of zero mean and unit variance. Suppose the particle starts from a position $q(t)=q_{0}$ close to the wall and its end position is in the wall. The regular auxiliary path is then parameterized by $q(t+\lambda \mathrm{d} t)=q_{0}+v \lambda \mathrm{d} t+\sqrt{2 D_{q} \mathrm{~d} t} \sqrt{\lambda} G$ with $\lambda \in[0,1]$ and fixed realization $G$. From this path a 'collision time' $\lambda_{0}$ with the wall can be calculated (note that $\lambda_{0}$ itself is a random variable). Then the particle is propagated along the path to the collision point corresponding to an elapsed time $\lambda_{0} \mathrm{~d} t$. Starting at the wall the particle is then propagated the remaining time $\mathrm{d} \tau=\mathrm{d} t-\lambda_{0} \mathrm{~d} t$ with a new realization of the Gaussian random variable such that the initial part of the path leads away from the surface and a new physical end position is obtained. This 

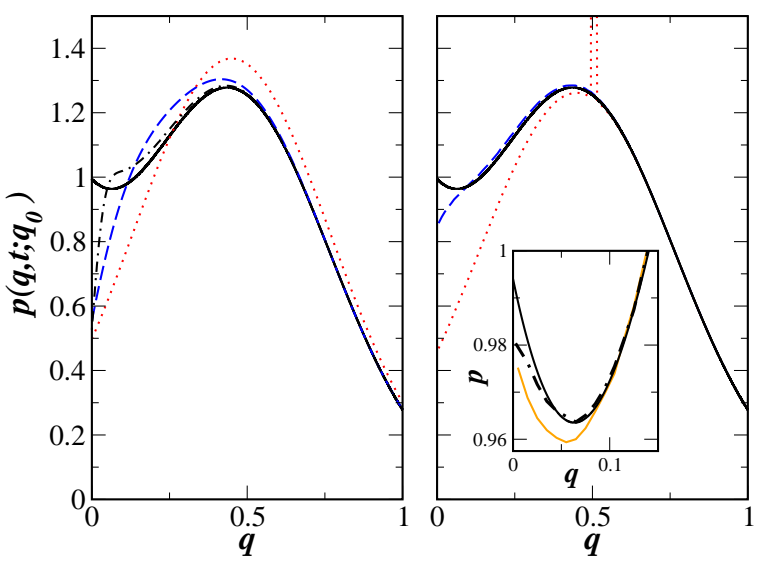

FIG. 1. Transition probability of a particle on a half-line for the parameters $v=-1.0, D_{q}=1.0$, initial position $q_{0}=0.5$ (corresponding to an encounter probability $w$, equation (10), of approximately $8 \%$ ) and elapsed time $t=0.05^{28}$ The analytical curve (6) is displayed by the solid line and is compared to results from numerical simulations based on the Euler algorithm using the rejection method without advancing time (left) and updating time (right). The (red) dotted curve is obtained with $\mathrm{d} t=0.05$. For the rejection scheme with updating time a delta-like peak at the initial position emerges representing the probability to encounter the wall. ${ }^{29}$ The (blue) dashed and the dotted-dashed curves were obtained using $\mathrm{d} t=0.005=t / 10$ and $\mathrm{d} t=0.0005=t / 100$, respectively. (For comparison the light (orange) line in the inset shows results from the event-driven method discussed below with $\mathrm{d} t=t=0.05$.)

approach obviously captures the influence of encounter events, however, the contributions from $p_{2}$ and $p_{3}$ are not completely collected. Suppose, for instance, that the deterministic force is pointing away from the wall, i.e. $v>0$. For a negative Gaussian variable $G$ the path for the propagation for the remaining time $\mathrm{d} \tau$ starting directly at the wall has an initial part which will always lead into the wall although it might produce a valid endposition. Such a path has to be rejected. A physical end position $q \in[0, \infty[$ is therefore only generated if the sign of the Gaussian random variable $G$ is positive. The physical end-position is therefore systematically shifted away from the wall by an amount of $\mathcal{O}(v \mathrm{~d} \tau)$. To avoid this systematic shift one can relax the conditions on the random Gaussian variable and allow all paths that lead to valid end-positions. We use this latter variant for the discussions throughout the present paper. In addition, the event-driven approach suffers from the serious drawback that the sampling of a probability distribution directly from trajectories never can generate the negative contributions of $p_{3}$ for forces $v>0$. Hence, a procedure that tries to incorporate the presence of a hard wall into a scheme that generates (discretized and regular approximations of the) trajectories will not correctly capture the contributions of $p_{3}$.

Figure 2 displays the analytical solution (6) of the
Fokker-Plank equation (5) together with transition probabilities obtained from the event-driven scheme for different initial separations $q_{0}$ from the wall. As expected, the systematic deviations are reduced for increasing $q_{0}$ for which the encounter probability is diminishing as well.

In principle the one-step distribution function for the event-driven scheme can be constructed analogously to (9) from $X=X_{\mathrm{G}} \theta\left(X_{\mathrm{G}}\right)+X_{\mathrm{K}} \theta\left(-X_{\mathrm{G}}\right)$ where $X_{\mathrm{K}}$ represents the random variable generated in case of an encounter. This variable is distributed according to

$$
p_{\mathrm{K}}(x ; \mathrm{d} \tau)=\frac{\exp \left(-\frac{(x-v \mathrm{~d} \tau)^{2}}{4 D_{q} \mathrm{~d} \tau}\right)}{\sqrt{\pi D_{q} \mathrm{~d} \tau}\left(1+\operatorname{erf}\left(\sqrt{\frac{\mathrm{d} \tau}{4 D_{q}} v}\right)\right)}, \quad x>0 .
$$

However, the time $\mathrm{d} \tau$ depends on the value of $X_{\mathrm{G}}<0$ of the first integration attempt and is determined by

$$
\begin{aligned}
X_{\mathrm{G}} & =q_{0}+v \mathrm{~d} t+\sqrt{2 D_{q} \mathrm{~d} t} G, \\
0 & =q_{0}+v(\mathrm{~d} t-\mathrm{d} \tau)+\sqrt{2 D_{q}(\mathrm{~d} t-\mathrm{d} \tau)} G,
\end{aligned}
$$

from which $G$ can be eliminated and the physical $\mathrm{d} \tau \in$ $[0, \mathrm{~d} t]$ be computed. The resulting distribution is thus

$$
p_{\text {ev }}(q)=p_{1}(q)+\int_{-\infty}^{0} \mathrm{~d} x p_{\mathrm{K}}(q ; \mathrm{d} \tau(x)) p_{\mathrm{G}}(x) .
$$

This expression, however, cannot be evaluated in closed form. Recall that the event-driven scheme produces a distribution of remaining propagation times $\mathrm{d} \tau \in[0, \mathrm{~d} t]$. Due to relations (21) and (22) this can be expressed in terms of unphysical $x \in]-\infty, 0]$ as is done in the integral in (23).

We already noted that the event-driven scheme includes effects due to encounter events with the wall. In particular, all events that encounter the wall precisely once are correctly captured whereas those with several encounters are approximated by trajectories with one encounter only. This observation readily suggests a refinement of the event-driven scheme. Once the particle is propagated to the first collision point the event-driven scheme is applied to the remaining time interval so that it is either propagated directly into the valid region or again a further collision point and collision time have to be determined. This approach is then iteratively applied till the particle is propagated for the total time step $\mathrm{d} t$. From numerical investigations (not shown in this article) we conclude that this iterative event-driven scheme indeed leads to correct transition probabilities for forces into the wall. For forces away from the wall, however, the procedure is not correct as the negative contribution from $p_{3}$ cannot be captured in this trajectory-based method.

We note at this point that Lamm and Schulten ${ }^{30}$ developed also a scheme which successively takes overlap events into account. If an overlap is detected a further displacement away from the wall is determined with a remaining propagation time that is sampled with the help 

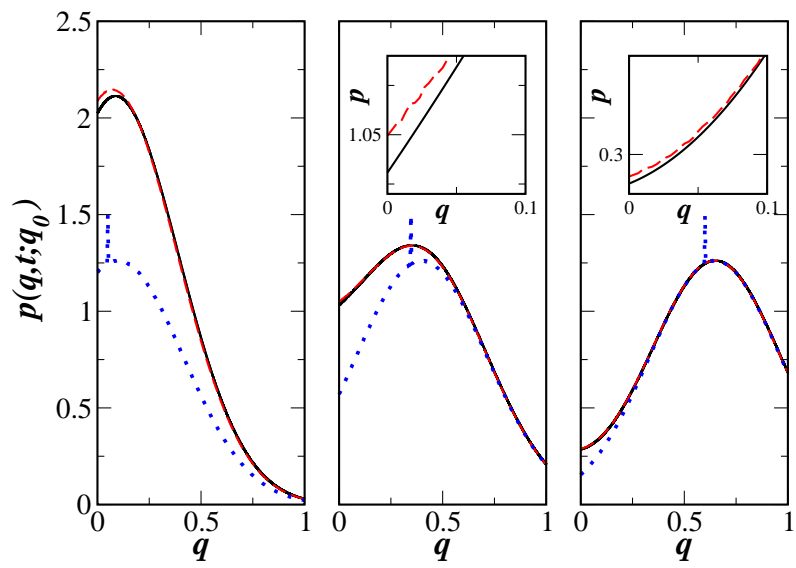

FIG. 2. Transition probability of a particle on the half-line for $v=1.0, D_{q}=1.0$ and elapsed time $t=0.05 .^{28}$ Three different initial positions $q_{0}$ are shown, namely, from left to right, $q_{0}=0.05$ (corresponding to a probability $w$ of approximately $38 \%$ to encounter the wall), $q_{0}=0.35(10 \%)$ and $q_{0}=0.6$ $(2 \%)$. The solid curves depict the analytical solutions (6). The numerical results are obtained by one integration step of the Euler scheme. The (red) dashed curves correspond to the event-driven method. For comparison the (blue) dotted line represents the results from the rejection method. In these curves a delta-like peak shows up at the initial position $q_{0}$ representing the particles that encounter the wall, and are thus not displaced in the rejection method (indicated by a spike). The insets show the deviation of the results from the event-driven scheme close to the wall, each with the same magnification.

of an approximation of the collision time distribution. If this still leads to an overlap a third step is generated according to (7c) approximated by a Boltzmann distribution. For details see Ref. 30 .

\section{Supplementary remarks}

Due to the presence of the hard wall (reflecting boundary) at the origin, the statistical properties of the particle displacements for finite propagation times $\mathrm{d} t$ deviate from a Gaussian (compare the Smoluchowski solution (6)). For a diffusing particle close to a wall, for instance, the first central moment $\left\langle q-q_{0}\right\rangle$ will not be equal to $v \mathrm{~d} t$ and thus will not vanish even for absent deterministic drift. Only in the limit $\sqrt{2 D_{q} \mathrm{~d} t} / q_{0} \rightarrow 0$ (and $q_{0}>0$ ) the 'Gaussian' statistics is restored, i. e. for small propagation time and/or sufficiently far away from the wall, so that its influence is practically not noticeable. For analyzing how well the different integrations schemes reproduce these deviations from a Gaussian behavior close to the wall, we quantify them by looking at the $n$th expansion coefficient of the Kramers-Moyal series $\underline{\underline{6}-\underline{8}}$ that is associated with the transition probability (6) of a diffus- ing particle on a half-line subject to a constant force:

$$
k_{n}\left(\mathrm{~d} t ; q_{0}\right)=\frac{1}{n ! \mathrm{d} t} \int_{-q_{0}}^{\infty} \mathrm{d} \zeta \zeta^{n} p\left(q_{0}+\zeta, \mathrm{d} t ; q_{0}\right) .
$$

We remark that relation (24) is used to estimate the drift term and the diffusion coefficient in FokkerPlanck or Langevin equations, respectively, from empirical data $\stackrel{31,32}{=}$ For $q_{0}>0$ one has $\lim _{\mathrm{d} t \rightarrow 0} k_{1}\left(\mathrm{~d} t ; q_{0}\right)=v$, $\lim _{\mathrm{d} t \rightarrow 0} k_{2}\left(\mathrm{~d} t ; q_{0}\right)=D_{q}$ and $\lim _{\mathrm{d} t \rightarrow 0} k_{n}\left(\mathrm{~d} t ; q_{0}\right)=0$ for all $n>2$, i. e. Gaussian statistics is recovered in the limit of vanishing time steps $\mathrm{d} t$. For finite $\mathrm{d} t$, the second and third coefficients are shown in Fig. 3 as a function of the initial separation from the wall for the different integration schemes. The moments exhibit a strong dependence on $q_{0}$ close to the wall and show a non-Gaussian behavior. For example, the third moment 'vanishes' only for considerable distances from the wall. In general, for increasing distance $q_{0}$ the coefficients approach the Gaussian values for unrestricted diffusion in a constant force field ('bulk-like' situation), namely $D_{q}+\frac{1}{2} v^{2} \mathrm{~d} t$ for $k_{2}$ and $D_{q} v \mathrm{~d} t+\mathcal{O}\left(\mathrm{d} t^{2}\right)$ for $k_{3}$.
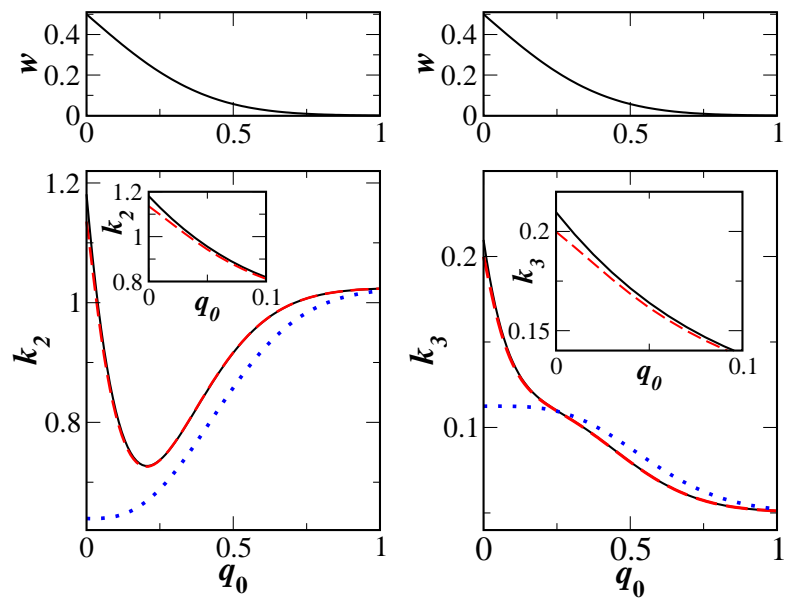

FIG. 3. Second and third ('finite-time') coefficient $k_{2}\left(\mathrm{~d} t ; q_{0}\right)$ (lower left panel) and $k_{3}\left(\mathrm{~d} t ; q_{0}\right)$ (lower right panel) of the Kramers-Moyal expansion as defined in (24) for a diffusing particle on a half-line with $v=1.0, D_{q}=1.0$ and elapsed time $\mathrm{d} t=0.05{ }^{28}$ The solid curves depict the $k_{n}$ from the analytical solution (6). The results are obtained using one integration step of the Euler scheme. The (red) dashed curves correspond to the event-driven method (using (23) and numerical integration), the (blue) dotted line represents the rejection method (with advancing time). The event-driven data is only slightly off the exact curve (compare insets). The upper graphs on both sides show the probability $w$ for an encounter with the wall, see relation (10).

From Fig. 3 we see that in particular close to the wall the rejection scheme shows significant deviations from the exact solution. Its convergence properties do not only depend on the choice of $\mathrm{d} t$ with respect to the force field, but also on how close a trajectory approaches the wall. 
Figure 4 illustrates this mutual dependence of a proper $\mathrm{d} t$ and the distance by analyzing how good the statistical properties of the stochastic evolution are captured. In contrast, the choice of $\mathrm{d} t$ for our algorithm is not influenced by the distance from the wall and is solely limited by the variations in the force field.

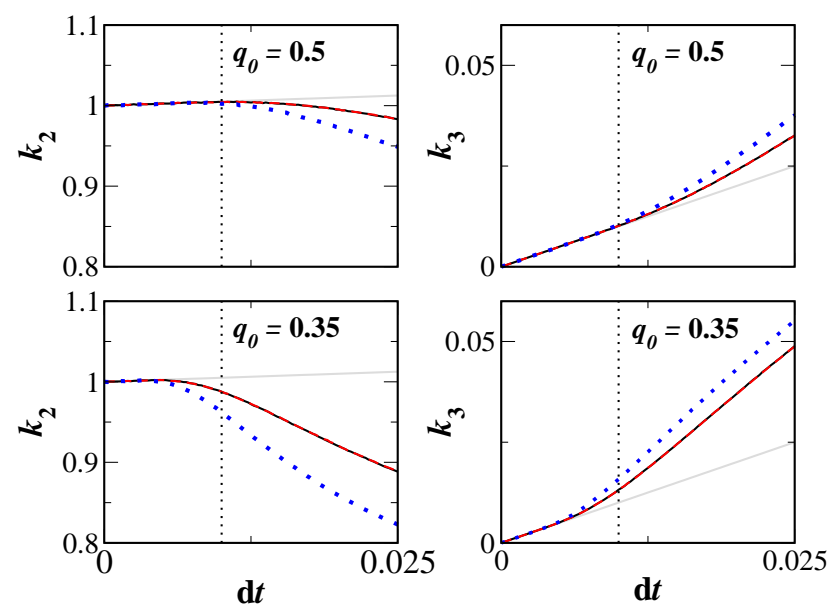

FIG. 4. Second and third ('finite-time') coefficient $k_{2}\left(\mathrm{~d} t ; q_{0}\right)$ (left hand side) and $k_{3}\left(\mathrm{~d} t ; q_{0}\right)$ (right hand side) of the Kramers-Moyal expansion as defined in (24) for the different algorithms as a function of the time step $\mathrm{d} t(v=1.0$, $\left.D_{q}=1.0\right)$ to illustrate their convergence. ${ }^{28}$ Suppose the particle to be propagated by $\mathrm{d} t$ is located at $q_{0}=0.5$. The coefficients $k_{2}$ and $k_{3}$ are displayed in the upper panels. The black curves represent the exact expressions (by construction identical to our algorithm), the (red) dashed curves the event-driven scheme and the (blue) dotted curves the rejection method. (The light grey curves show the asymptotic expressions for $\mathrm{d} t \rightarrow 0$, 'bulk-like' situation, see main text). For the choice $\mathrm{d} t=0.01$ (vertical dashed lines) $k_{2}$ and $k_{3}$ are well reproduced by the rejection scheme. Suppose now that the particle is propagated to $q=0.35$. The coefficients for the next time step starting then at $q_{0}=0.35$ are displayed in the lower panels. Now, the rejection scheme shows considerable deviation in $k_{2}$ and $k_{3}$ for the originally adjusted $\mathrm{d} t=0.01$.

\section{HARD WALLS}

Our novel procedure to incorporate hard-body interactions into a Brownian dynamics simulation is now developed for space-fixed walls. The walls might be curved so that their normal vectors attain a position dependence. The discussion will be carried out for point like Brownian particles. For extended spherical particles the distance to the hard surface is reduced by the radius of the particle.

To develop the method independently of the used algorithm for the numerical integration we discuss the procedure on the level of the Langevin equation (2) formulated in terms of differentials with the tacit understanding that $\mathrm{d} \vec{r}$ has to be seen as the numerically obtained displacement for the (discrete) time step $\mathrm{d} t$. We also assume that the time step is already chosen appropriately small to account for variations in the force fields.

\section{A. Description of algorithm}

Suppose that the Brownian particle is currently at position $\vec{r}$ close to the wall and experiences the deterministic force $\vec{F}$. Then, the standard integration scheme, which 'suggests' a displacement $\mathrm{d} \vec{r}$, is modified in the following way to yield a physically valid displacement $\mathrm{d} \vec{r}^{*}$, which accounts for the hard wall:

1. Calculate a 'preliminary' displacement $\mathrm{d} \vec{r}$ using the standard integrator for the 'free' Langevin equation without wall.

2. Check whether or not the so suggested new position $\vec{r}+\mathrm{d} \vec{r}$ yields a physically valid configuration (i.e. whether or not the particle, if at position $\vec{r}+\mathrm{d} \vec{r}$, overlaps with the hard wall).

a. If this configuration is valid, the suggested displacement is accepted as the new one, $\mathrm{d} \vec{r}^{*}=$ $\mathrm{d} \vec{r}$.

b. If this configuration is invalid, the new, valid displacement is calculated according to

$$
\mathrm{d} \vec{r}^{*}=\mathrm{d} \vec{r}+\left(q-q_{0}-\vec{n} \cdot \mathrm{d} \vec{r}\right) \vec{n},
$$

where $\vec{n}$ is the normal on the wall that passes through $\vec{r}, q_{0}$ is the distance of the particle at initial position $\vec{r}$ from the wall, and $q$ is generated from $p_{\mathrm{H}}\left(q, q_{0}, \mathrm{~d} t, v, D_{q}\right)$ with $v=\vec{n}$. $\vec{F} / \eta$ and $D_{q}=D$ (compare relation (8) and Appendix A).

We note that $\mathrm{d} t$ has to be adjusted such that $\lambda \gg \mathcal{O}(|\mathrm{d} \vec{r}|)$ holds true with $\lambda$ being the smallest radius of curvature of the wall.

\section{B. Justification and discussion}

For the time step that has produced the unphysical configuration the displacement can be decomposed into a displacement perpendicular to the wall (along the normal $\vec{n})$,

$$
\mathrm{d} \vec{r}_{\perp}=(\vec{n} \cdot \mathrm{d} \vec{r}) \vec{n}=\frac{\vec{F}_{\perp}}{\eta} \mathrm{d} t+\sqrt{2 D} \mathrm{~d} \vec{W}_{\perp},
$$

and a displacement parallel to the wall,

$$
\mathrm{d} \vec{r}_{\|}=\mathrm{d} \vec{r}-(\vec{n} \cdot \mathrm{d} \vec{r}) \vec{n}=\frac{\vec{F}_{\|}}{\eta} \mathrm{d} t+\sqrt{2 D} \mathrm{~d} \vec{W}_{\|} .
$$

The perpendicular component of the deterministic force is given by $\vec{F}_{\perp}=(\vec{n} \cdot \vec{F}) \vec{n}$ whereas the parallel component is $\vec{F}_{\|}=\vec{F}-\vec{F}_{\perp}$. The components of the fluctuating force are obtained correspondingly and are given by 
$\mathrm{d} \vec{W}_{\perp}=(\mathrm{d} \vec{W} \cdot \vec{n}) \vec{n}$ and $\mathrm{d} \vec{W}_{\|}=\mathrm{d} \vec{W}-(\mathrm{d} \vec{W} \cdot \vec{n}) \vec{n}$. These two components are uncorrelated due to the orthonormality of the perpendicular and parallel directions:

$$
\begin{aligned}
\left\langle\mathrm{d} W_{\perp}^{\alpha} \mathrm{d} W_{\|}^{\beta}\right\rangle & =\left\langle\mathrm{d} W^{\mu} n^{\mu} n^{\alpha}\left(\mathrm{d} W^{\beta}-\mathrm{d} W^{\nu} n^{\nu} n^{\beta}\right)\right\rangle \\
& =\mathrm{d} t n^{\alpha} n^{\beta}\left(1-n^{\mu} n^{\mu}\right)=0 .
\end{aligned}
$$

The correlations need to be considered only for the current time step where an unphysical position is generated. The normal $\vec{n}$ sets up a locally fixed coordinate system. However, one is not interested in its dynamical properties so that its (possible) position dependence has not to be considered in a dynamical (co-moving) sense. For this particular time step the random forces in the two equations are those that appear at time $t$, for the corresponding diffusion constants one has $D_{\perp}=D_{\|}=D$. It is essential that the perpendicular and parallel components of the displacement decouple and decorrelate for this local coordinate, so that they can be considered independently.

Let us assume for the moment that we have an appropriately small time step so that the typical displacement $\mathrm{d} \vec{r}$ is small compared to the (smallest) radius of curvature of the point on the wall that corresponds to the normal $\vec{n} \underline{\underline{33}}$ Hence, the wall appears to be flat on the scale of $\mathrm{d} \vec{r}$. In this approximation, the perpendicular motion along the normal $\vec{n}$ thus corresponds to a motion on an infinite half-line, whereas the motion along the parallel direction is unrestricted. The overlap with the wall then solely results from the (one-dimensional) displacement along $\vec{n}$. A valid physical end position along that direction, i. e. the distance $q$ from the wall after elapsed time $\mathrm{d} t$, is now directly generated from (8), with $q_{0}$ being the separation of the initial position $\vec{r}$ from the wall, $D_{q}=D$ and $v=\vec{n} \cdot \vec{F} / \eta$.

The parallel motion is uncorrelated with the perpendicular motion for the considered time step on the level of the fluctuating forces and therefore is unaffected by that modification. We note that the deterministic force is fixed for the considered time step and that the modification accounts for the altered statistical properties of the random forces in the vicinity of a hard wall. Hence, the already proposed displacement $\mathrm{d} \vec{r}_{\|}$can be retained to avoid unnecessary computational efforts. The total modified physical displacement is then given by $\mathrm{d} \vec{r}^{*}=$ $\mathrm{d} q \vec{n}+\mathrm{d} \vec{r}_{\|}$with $\mathrm{d} q=q-q_{0}$. Plugging in the originally proposed $\mathrm{d} \vec{r}$ on gets the modified $\mathrm{d} \vec{r}^{*}=\mathrm{d} \vec{r}+(\mathrm{d} q-\vec{n} \cdot \mathrm{d} \vec{r}) \vec{n}$ already given above.

We note that the maximum allowable size of the integration time step $\mathrm{d} t$ is limited by the curvature of the hard wall. However, the length scales of physical systems for which Brownian dynamics is applied is typically mesoscopic so that walls are only weakly curved on the scale of typical displacements and thus the corresponding radius of curvature is large. To be more explicit, consider protein-protein association as a paradigmatic example. Here the extension of (space-fixed) obstacles is usually comparable to (or even larger than) the diameter of the proteins themselves, say $40 \AA$. The range of the involved non-covalent forces is typically of the order of 2 to $4 \AA, \underline{54,35}$ Therefore, the time step that has to be used to capture the variations in the force fields is already small enough to account for the curvature of the obstacles as well. In summary, the local flatness of the boundary is necessary for our algorithm to be applicable, but this approximation can be controlled by the choice of the size of the time step very much in the same way as space-dependent variations of the forces are captured by sufficiently small time steps.

Finally, we want to point out that in the force-free case $v=0$, the solution (6) simplifies to $p\left(q, t ; q_{0}\right)=$ $p_{1}\left(q, t ; q_{0}\right)+p_{1}\left(q, t ;-q_{0}\right)\left(\right.$ since $p_{2}\left(q, t ; q_{0}\right)=p_{1}\left(q, t ;-q_{0}\right)$ and $p_{3}\left(q, t ; q_{0}\right)=0$, compare (77) $)$, consistent with what one would obtain from the so-called image method (see also Appendix B). As a consequence, our new algorithm, which is constructed from expressions (6) and (7), becomes equivalent (in a strict mathematical sense) to numerical schemes which replace invalid positions by an image end-point whenever a wall is encountered and externally applied forces are absent. A prominent example is the algorithm developed by Scala et al. $\stackrel{17}{ }$

\section{HARD SPHERES}

In the previous section physical systems with spacefixed hard walls have been considered. In many systems, however, movable objects with hard cores are present as well. In this section we describe, how the previously discussed method can be extended to encounters of two hard spheres, which both move under the influence of deterministic forces and diffusion.

\section{A. Description of algorithm}

Consider two hard spheres with (possibly different) radii $a_{i}$, friction coefficients $\eta_{i}$ and diffusion constants $D_{i}=k_{\mathrm{B}} T / \eta_{i}$ whose dynamics is described by the two Langevin equations

$$
\mathrm{d} \vec{r}_{1}=\vec{v}_{1} \mathrm{~d} t+\sqrt{2 D_{1}} \mathrm{~d} \vec{W}_{1}
$$

and

$$
\mathrm{d} \vec{r}_{2}=\vec{v}_{2} \mathrm{~d} t+\sqrt{2 D_{2}} \mathrm{~d} \vec{W}_{2} .
$$

Here again the drift terms are denoted in terms of velocities $\vec{v}_{i}=\vec{F}_{i}\left(\vec{r}_{i}, t\right) / \eta_{i}$ for the two particles.

Suppose now that the two spheres are at $\vec{r}_{1}$ and $\vec{r}_{2}$ at time $t$ and that we have at hand a standard integration scheme to update the particle positions for a time step $\mathrm{d} t$. Then, the algorithm for generating valid displacements $\mathrm{d} \vec{r}_{1}^{*}$ and $\mathrm{d} \vec{r}_{2}^{*}$ consistent with hard-wall interactions between these two particles is as follows:

1. Use the standard integration scheme to generate 'preliminary' propagation steps $\mathrm{d} \vec{r}_{1}, \mathrm{~d} \vec{r}_{2}$. 
2. Check whether or not the suggested end positions $\vec{r}_{1}+\mathrm{d} \vec{r}_{1}$ and $\vec{r}_{2}+\mathrm{d} \vec{r}_{2}$ represent a physically valid configuration, i. e. whether or not the two spheres at positions $\vec{r}_{1}+\mathrm{d} \vec{r}_{1}$ and $\vec{r}_{2}+\mathrm{d} \vec{r}_{2}$ would overlap.

a. If the two spheres do not overlap, the new configuration is accepted, $\mathrm{d} \vec{r}_{1}^{*}=\mathrm{d} \vec{r}_{1}$ and $\mathrm{d} \vec{r}_{1}^{*}=\mathrm{d} \vec{r}_{1}$.

b. If the two spheres do overlap, valid displacements $\mathrm{d} \vec{r}_{1}^{*}, \mathrm{~d} \vec{r}_{2}^{*}$ are constructed according to the following procedure:

- Calculate the separation $q_{0}=\left|\vec{r}_{2}-\vec{r}_{1}\right|-$ $\left(a_{1}+a_{2}\right)$ of the surfaces of the two spheres and determine the normalized connection vector $\vec{e}=\left(\vec{r}_{2}-\vec{r}_{1}\right) /\left|\vec{r}_{2}-\vec{r}_{1}\right|$.

- Generate a position $q$ from $p_{\mathrm{H}}\left(q, q_{0}, \mathrm{~d} t, v, D_{q}\right)$ with diffusion constant $D_{q}=D_{1}+D_{2}$ and velocity $v=\left(\vec{v}_{2}-\vec{v}_{1}\right) \cdot \vec{e}($ compare relation (8) and Appendix A).

- Use $\mathrm{d} q=q-q_{0}$ to evaluate the modified physical displacements

$$
\mathrm{d} \vec{r}_{1}^{*}=\mathrm{d} \vec{r}_{1}+\frac{\eta_{2}}{\eta_{1}+\eta_{2}}\left(\left(\mathrm{~d} \vec{r}_{2}-\mathrm{d} \vec{r}_{1}\right) \cdot \vec{e}-\mathrm{d} q\right) \vec{e}
$$

and

$$
\mathrm{d} \vec{r}_{2}^{*}=\mathrm{d} \vec{r}_{2}-\frac{\eta_{1}}{\eta_{1}+\eta_{2}}\left(\left(\mathrm{~d} \vec{r}_{2}-\mathrm{d} \vec{r}_{1}\right) \cdot \vec{e}-\mathrm{d} q\right) \vec{e} .
$$

The time step $\mathrm{d} t$ has to by chosen small enough so that $a_{1}+a_{2} \gg \mathcal{O}\left(\left|\mathrm{d} \vec{r}_{2}-\mathrm{d} \vec{r}_{1}\right|\right)$ is satisfied.

\section{B. Justification and discussion}

To generate a physical configuration without overlap the motion of the two Brownian particles is decomposed into a common center-of-friction motion and a relative motion of the two spheres. The center-of-friction $\vec{R}=$ $\left(\eta_{1} \vec{r}_{1}+\eta_{2} \vec{r}_{2}\right) / \eta, \eta=\eta_{1}+\eta_{2}$, obeys the Langevin equation

$$
\begin{aligned}
\mathrm{d} \vec{R}= & \frac{1}{\eta}\left(\eta_{1} \vec{v}_{1}+\eta_{2} \vec{v}_{2}\right) \mathrm{d} t \\
& +\frac{1}{\eta}\left(\eta_{1} \sqrt{2 D_{1}} \mathrm{~d} \vec{W}_{1}+\eta_{2} \sqrt{2 D_{2}} \mathrm{~d} \vec{W}_{2}\right) \\
= & \vec{V} \mathrm{~d} t+\sqrt{2 D_{R}} \mathrm{~d} \vec{W}_{R}
\end{aligned}
$$

and the relative motion of $\vec{r}=\vec{r}_{2}-\vec{r}_{1}$ is governed by

$$
\begin{aligned}
\mathrm{d} \vec{r} & =\left(\vec{v}_{2}-\vec{v}_{1}\right) \mathrm{d} t+\sqrt{2 D_{2}} \mathrm{~d} \vec{W}_{2}-\sqrt{2 D_{1}} \mathrm{~d} \vec{W}_{1} \\
& =\vec{v} \mathrm{~d} t+\sqrt{2 D_{r}} \mathrm{~d} \vec{W}_{r} .
\end{aligned}
$$

Here, the abbreviations $\vec{v}:=\vec{v}_{2}-\vec{v}_{1}$ and $\vec{V}:=$ $\frac{1}{\eta}\left(\eta_{1} \vec{v}_{1}+\eta_{2} \vec{v}_{2}\right)=\frac{1}{\eta}\left(\vec{F}_{1}+\vec{F}_{2}\right)$ have been introduced. The fluctuation terms in (32) and (34) can again be written in terms of un-correlated white noise terms $\mathrm{d} \vec{W}_{R}$ and $\mathrm{d} \vec{W}_{r}$ with associated diffusion coefficients $D_{R}=$
$k_{\mathrm{B}} T / \eta=D_{1} D_{2} /\left(D_{1}+D_{2}\right)$ and $D_{r}=D_{1}+D_{2}$, respectively. The statistical properties of the fluctuation terms in (32) and (34) are readily obtained from the observation that the sum and the difference of two Wiener processes are again Wiener processes (as theses processes are Gaussian stochastic processes with vanishing mean). Due to the appropriate weighting of the contributions of the two particles in the definition of the center-of-friction, $\mathrm{d} \vec{W}_{R}$ and $\mathrm{d} \vec{W}_{r}$ are uncorrelated, as can be seen directly by equating the cross correlations.

As a consequence, any corrections in the relative motion do not affect the propagation in center-of-friction $\vec{R}$ (and vice versa). The overlap of the two particles is the result of the unphysical propagation in the relative coordinate $\vec{r}$ so that this very motion has to be modified due to the hard-wall interaction, while the initially proposed displacement $\mathrm{d} \vec{R}$ of the center-of-friction can be kept to avoid unnecessary computational efforts. This is similar to the situation of a particle in the vicinity of a wall where the normal and the parallel displacements decorrelate.

The relative motion itself corresponds to the motion of a point-particle close to a space-fixed sphere of radius $a_{1}+a_{2}$. The treatment of the relative motion therefore follows the lines of reasoning already developed in Sec. IV] The relative displacement $\mathrm{d} \vec{r}$ can be further decomposed into a (one-dimensional) displacement $\mathrm{d} \vec{r}_{\perp}$ along the connection line defined by the unit vector $\vec{e}:=\vec{r} /|\vec{r}|$ and a displacement $\mathrm{d} \vec{r}_{\|}$in the plane perpendicular to $\vec{e}$. Thus one obtains the equations

$$
\mathrm{d} r_{\perp}=\vec{v} \cdot \vec{e} \mathrm{~d} t+\sqrt{2 D_{r}} \mathrm{~d} \vec{W}_{r} \cdot \vec{e}
$$

and

$$
\mathrm{d} \vec{r}_{\|}=\left(\vec{v}-\vec{v}_{\perp}\right) \mathrm{d} t+\sqrt{2 D_{r}}\left(\mathrm{~d} \vec{W}_{r}-\left(\mathrm{d} \vec{W}_{r} \cdot \vec{e}\right) \vec{e}\right)
$$

with $\vec{v}_{\perp}=(\vec{e} \cdot \vec{v}) \vec{e}$. (Note that $\vec{e}$ is the normal of the effective hard surface that restricts the motion of the relative coordinate. The nomenclature of perpendicular and parallel displacement has to be seen with respect to this spherical surface). The crucial point is that the perpendicular fluctuating term $\mathrm{d} W_{\perp}=\mathrm{d} \vec{W}_{r} \cdot \vec{e}$ and the parallel random term $\mathrm{d} \vec{W}_{\|}=\mathrm{d} \vec{W}_{r}-\left(\mathrm{d} \vec{W}_{r} \cdot \vec{e}\right) \vec{e}$ are again un-correlated (for the current time step where the overlap is detected). The associated diffusion constants are $D_{\perp}=D_{\|}=D_{r}$. The vector $\vec{e}$ is fixed for the considered time step and again defines a local coordinate system suitable for the decomposition.

Assuming that the effective hard wall (that corresponds to a sphere of radius $a_{1}+a_{2}$ ) can be well approximated as flat (which is, for example, well fulfilled in the typical case in point of protein-protein association, see Sec. IV], the relative motion along the direction $\vec{e}$ then corresponds to the motion on an infinite halfline and the parallel motion is unrestricted in an infinite plane. Therefore, the described algorithm of Sec. II for the one-dimensional system can be applied directly to generate a new physical position along $\vec{e}$. The propagation in the plane perpendicular to $\vec{e}$ is not affected 
by that modification and thus the corresponding initially obtained displacement can be kept. To satisfy the assumption that the hard surface is virtually flat the time step has to be chosen sufficiently small so that one has $a_{1}+a_{2} \gg \mathcal{O}(|\mathrm{d} \vec{r}|) \cdot 33$

The new configuration without overlap of the spheres is obtained from the modified relative displacement by

$$
\mathrm{d} \vec{r}^{*}=\mathrm{d} \vec{r}_{\|}+\mathrm{d} q \vec{e}
$$

with $\mathrm{d} \vec{r}_{\|}=\mathrm{d} \vec{r}-(\mathrm{d} \vec{r} \cdot \vec{e}) \vec{e}$ being the originally proposed parallel displacement ( $\mathrm{d} \vec{r}$ denotes the initially proposed relative displacement $\mathrm{d} \vec{r}_{2}-\mathrm{d} \vec{r}_{1}$ of the two spheres). Here, $\mathrm{d} q=q-q_{0}$ is fixed by $q$ that is generated from distribution (8) with diffusion constant $D_{q}=D_{\perp}=D_{1}+D_{2}$, velocity $v=\vec{v} \cdot \vec{e}=\left(\vec{v}_{2}-\vec{v}_{1}\right) \cdot \vec{e}$, initial separation $q_{0}=\left|\vec{r}_{2}-\vec{r}_{1}\right|-\left(a_{1}+a_{2}\right)$ of the two spheres. Once $\mathrm{d} \vec{r}^{*}$ is determined the new absolute particle positions without overlap are calculated from the new displacements as

$$
\mathrm{d} \vec{r}_{1}^{*}=\mathrm{d} \vec{R}-\frac{\eta_{2}}{\eta_{1}+\eta_{2}} \mathrm{~d} \vec{r}^{*}
$$

and

$$
\mathrm{d} \vec{r}_{2}^{*}=\mathrm{d} \vec{R}+\frac{\eta_{1}}{\eta_{1}+\eta_{2}} \mathrm{~d} \vec{r}^{*}
$$

Plugging in the explicit expressions for the various quantities one ends up with equations (30a) and (30b).

\section{EXEMPLARY MODEL SYSTEMS}

In this section we numerically investigate the applicability and quality of the new algorithm in comparison with the heuristic methods of Sec. III. We do not aim at a study of particular physical systems or phenomena, we rather intend to present exemplary problems that typically have to be dealt with in systems modelled within Brownian dynamics. To set up an explicit frame we look at two research areas from soft matter, namely the motion of colloids in flow fields and the interaction of proteins in solution. For simplicity we consider only two-dimensional systems. All numerical results presented below are obtained by using the Euler algorithm as standard integrator.

\section{A. Colloids in flow fields}

The first characteristic system we look at is given by colloidal particles in stationary flow fields $\vec{u}(\vec{r})$. To begin with we consider a spherical particle of radius $a=1 \mu \mathrm{m}$ and with diffusion constant $D=0.21 \mu \mathrm{m}^{2} / \mathrm{s}$ (StokesEinstein diffusion at room temperature $k_{\mathrm{B}} T_{\text {room }} \approx$ $4 \mathrm{fN} \mu \mathrm{m}$ ) suspended in the fluid flow around a space-fixed circular post in two space dimensions. This model system is taken to mimic a typical situation in microfluidic devices where particles encounter posts or walls in the channel. Let us consider a flow field with a typical velocity of $u_{0}=10 \mu \mathrm{m} / \mathrm{s}$ (typical velocities in microfluidic channels range from $1 \mu \mathrm{m} / \mathrm{s}$ to $1 \mathrm{~cm} / \mathrm{s}$ ) around a circular post of radius $R=10 \mu \mathrm{m}$ at the origin of the coordinate system. For a time step $\mathrm{d} t=0.01 \mathrm{~s}$ the typical diffusive displacement $\sqrt{\left\langle\vec{r}^{2}\right\rangle-\langle\vec{r}\rangle^{2}}$ of a particle with radius $a=1 \mu \mathrm{m}$ is $0.1 \mu \mathrm{m}$. Thus we deduce that for a radius of $R=10 \mu \mathrm{m}$ of the post the curvature of the hard surface should not limit the applicability of our method. Assuming no-slip boundary conditions, the velocity field of the two-dimensional stationary Stokes flow $\vec{u}(\vec{r})=\left(u_{x}(x, y), u_{y}(x, y)\right)$ around the post is given by

$$
u_{x}=u_{0} \frac{y^{2}-x^{2}}{x^{2}+y^{2}}\left(1-\frac{R^{2}}{x^{2}+y^{2}}\right)+2 u_{0} \ln \frac{\sqrt{x^{2}+y^{2}}}{R}
$$

and

$$
u_{y}=-u_{0} \frac{x y}{x^{2}+y^{2}}\left(1-\frac{R^{2}}{x^{2}+y^{2}}\right)
$$

in Cartesian coordinates (for a discussion of the Stokes approximation for small Reynolds numbers and its validity in two space dimensions, see for example Refs. 37 and 38).
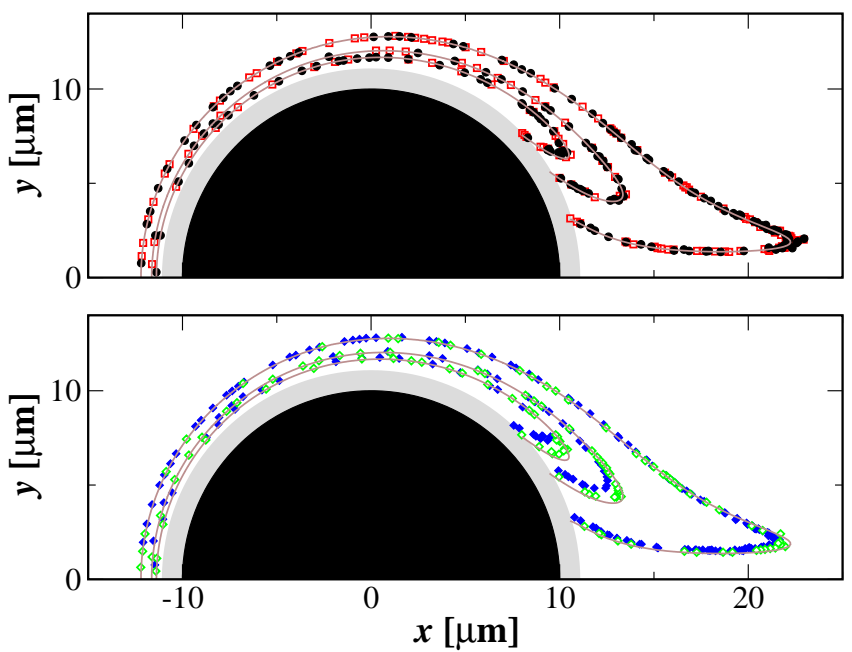

FIG. 5. Contour plot of the distribution of a spherical colloid (radius $a=1 \mu \mathrm{m}$, diffusion constant $D=0.21 \mu \mathrm{m}^{2} / \mathrm{s}$ ) flowing around a circular obstacle of radius $R=10 \mu \mathrm{m}$. The particle starts initially at $\vec{r}(0)=(-11.5 \mu \mathrm{m}, 0 \mu \mathrm{m})$ and evolves for $t=10 \mathrm{~s}$. The Stokesian flow (38) is characterized by $u_{0}=10 \mu \mathrm{m} / \mathrm{s}$. The solid line represents the solution of the associated Fokker-Planck equation. In the upper panel symbols display the numerical solutions obtained from integrating the Langevin equation with $\vec{v}(\vec{r})=\vec{u}(\vec{r})$ for a time step $\mathrm{d} t=0.01 \mathrm{~s}$ with circles corresponding to our algorithm and open (red) squares to the event-driven scheme. The lower panel shows the data from the rejection scheme for $\mathrm{d} t=0.01 \mathrm{~s}$ (blue diamonds) and $\mathrm{d} t=0.001 \mathrm{~s}$ (open green diamonds). The black region represents the obstacle, the grey shaded region is inaccessible to the particle coordinate.

Figure 5 compares the numerically determined distribution function of a colloid in flow field (38) with the 
solution of the associated Fokker-Planck equation. The evolution of the particle as exhibited by the rejection scheme lags behind the expected behavior. To analyze this further we look at the mean first passage time the colloid needs to cross a certain threshold after passing the post. Figure 6 displays the corresponding results as a function of the integration step $\mathrm{d} t$. It shows also the
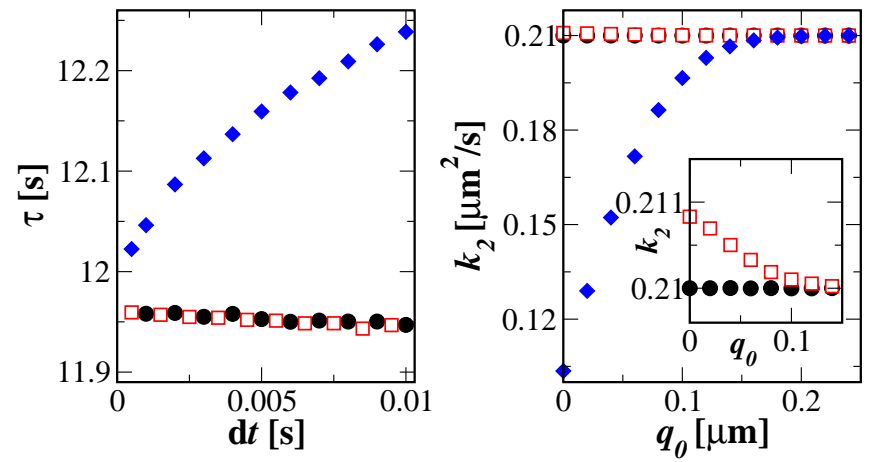

FIG. 6. Left panel: Mean first passage time $\tau$ a colloid of radius $a=1 \mu \mathrm{m}$ needs to flow past a post of radius $R=$ $10 \mu \mathrm{m}$ to the threshold at $x=b=11.5 \mu \mathrm{m}$ as a function of the integration time step. The colloid starts initially at $\vec{r}(0)=(-11.5 \mu \mathrm{m}, 0 \mu \mathrm{m})$. Our method is displayed by (black) circles, the event-driven scheme by (red) open squares and the rejection scheme by (blue) diamonds. For the numerical determination of the mean first passage time, see e.g. Refs. 9 and 36. Note that the collision frequency with the post drops from $3 \%$ to $1 \%$ for the shown range of $\mathrm{d} t$. Right panel and inset: Tangential diffusion $k_{2}=\left\langle\left(\mathrm{d} \vec{r} \cdot \vec{e}_{\|}\right)^{2}\right\rangle /(2 \mathrm{~d} t)$ during the first time step of size $\mathrm{d} t=0.01 \mathrm{~s}$ as a function of the initial distance $q_{0}=|\vec{r}(0)-(R+a)|$ between the surfaces of the colloid and the post. The different schemes are displayed using the symbol code of the left panel. The expected value is $0.21 \mu \mathrm{m}^{2} / \mathrm{s}$.

particle diffusion $k_{2}=\left\langle\left(\mathrm{d} \vec{r} \cdot \overrightarrow{e_{\|}}\right)^{2}\right\rangle /(2 \mathrm{~d} t)$ tangential to the post during the first time step as a function of the initial separation from the post's surface $\left(\vec{e}_{\|}\right.$denotes the tangential unit vector to the surface). The tangential motion is not restricted by the presence of the hard surface so that $k_{2}$ is expected to be given by the diffusion constant $D$ of the particle. For the rejection scheme, however, the tangential diffusion close to the wall is clearly reduced. This has the effect that the time the particle needs to flow around the obstacle is increased when measured from the rejection scheme. As can be seen in Fig. 6] this effect is reduced for decreasing time steps as the collision frequency with the obstacle goes down as well.

As a second typical case study we investigate the evolution of two colloidal particles in a prescribed shear flow, The velocity field at $\vec{r}=(x, y)$ is given by

$$
\vec{u}(\vec{r})=\alpha y \vec{e}_{x}
$$

where $\alpha$ is the shear rate and $\vec{e}_{x}$ denotes the unit vector in $x$-direction. Figure 7 depicts the distribution function of the relative coordinate of the two colloids for different elapsed times. Again numerical data is compared to the solution of the Fokker-Planck equation.
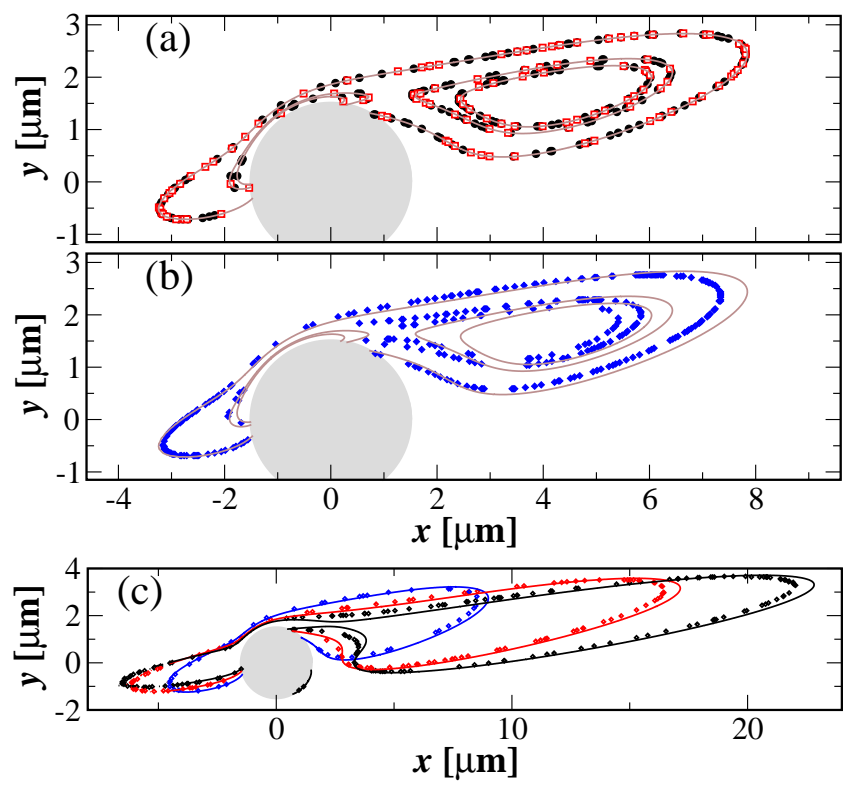

FIG. 7. Contour plot of the transition probability of the relative vector $\vec{r}=(x, y)=\vec{r}_{2}-\vec{r}_{1}$ of two colloids in a shear flow (39) with shear rate $\alpha=10 / \mathrm{s}$. The two particles with radii $a_{1}=1 \mu \mathrm{m}, a_{2}=0.5 \mu \mathrm{m}$ and friction coefficients $\eta_{1}=18.9 \mathrm{fNs} / \mu \mathrm{m}, \eta_{2}=9.45 \mathrm{fNs} / \mu \mathrm{m}$ (i.e. $D_{1}=0.21 \mu \mathrm{m}^{2} / \mathrm{s}$, $D_{2}=0.42 \mu \mathrm{m}^{2} / \mathrm{s}$ at room temperature) initially start at $\vec{r}_{1}(0)=(0 \mu \mathrm{m}, 0 \mu \mathrm{m})$ and $\vec{r}_{2}(0)=(-2 \mu \mathrm{m}, 0.75 \mu \mathrm{m})$, and evolve for $t=0.5 \mathrm{~s}$ (panels (a) and (b)). The solution of the associated Fokker-Planck equations are depicted by solid lines, the numerical results from integrating the Langevin equations with $\mathrm{d} t=0.002 \mathrm{~s}$ are shown by symbols: (Black) circles represent our algorithm, open (red) squares correspond to the event-driven scheme (panel (a)), and (blue) diamonds to the rejection approach (panel (b)). Panel (c) shows the evolution of one contour line with time $(0.5,0.8,1.0 \mathrm{~s})$ in comparison to the results of the rejection scheme. The grey circle represents the region which is inaccessible to the relative coordinate of the two colloids.

\section{B. Protein-protein interaction}

A (fairly small) protein has a typical radius of approximately $a=2 \mathrm{~nm}$ leading to a diffusion constant $D=$ $0.1 \mathrm{~nm}^{2} / \mathrm{ns}$ at room temperature $k_{\mathrm{B}} T_{\text {room }} \approx 4 \mathrm{pNnm}$. Therefore, the typical time a diffusing protein needs to cross its size is of the order of 10 ns. The forces between two proteins are governed by non-covalent interactions which have a typical energy scale of 2 to $4 k_{\mathrm{B}} T_{\text {room }}$ and a range of 0.2 to $0.4 \mathrm{~nm}$. Therefore, the velocity in the Langevin equation (2) is of the order of $5 \mathrm{~nm} / \mathrm{ns}$. A time step of $\mathrm{d} t=0.001 \mathrm{~ns}$ has a typical diffusive displacement $\sqrt{\left\langle\vec{r}^{2}\right\rangle-\langle\vec{r}\rangle^{2}}$ of approximately $0.02 \mathrm{~nm}$ and should be small enough to capture typical variations in the force 
fields $(\mathcal{O}(|\vec{v} \mathrm{~d} t|) \sim 0.005 \mathrm{~nm})$ and to resolve the details of trajectories.

To have an explicit example at hand we will look at the interaction of proteins such as lysozyme, which has a radius of $1.8 \mathrm{~nm}$ and a diffusion constant of $0.12 \mathrm{~nm}^{2} / \mathrm{ns}$ in aqueous solution. The force between two proteins stems from repulsive (screened) electrostatic and from attractive short-ranged van-der Waals interactions. Modelling the lysozyme solution as a colloidal system 39,40 within the Derjaguin-Landau-Verwey-Overbeek theory the electrostatic interaction between two proteins of separation $r=\left|\vec{r}_{2}-\vec{r}_{1}\right|$ is represented by the Debye-Hückel contribution

$$
\phi_{\mathrm{DH}}(r)=\frac{K}{r} \exp (-\kappa(r-\sigma))
$$

and the attractive van-der Waals part by

$$
\phi_{\mathrm{HA}}(r)=-\frac{A}{12}\left(\frac{\sigma^{2}}{r^{2}}+\frac{\sigma^{2}}{r^{2}-\sigma^{2}}+2 \ln \left(\frac{r^{2}-\sigma^{2}}{r^{2}}\right)\right) .
$$

The total interaction potential is then given by

$$
\phi(r)= \begin{cases}\phi_{\mathrm{DH}}(r)+\phi_{\mathrm{HA}}(r), & \text { if } r \geq \sigma+\delta \\ \infty, & \text { if } r<\sigma+\delta\end{cases}
$$

from which one obtains the force $\vec{F}_{i}=-\nabla_{i} \phi(r)$ on particle $i=1,2$. Here $\kappa$ denotes the screening length, $\sigma$ is the radius of the effective particle (i. e. the sum of the two radii) and $\delta$ is the Stern layer thickness so that the excluded hard-core radius is given by $R_{\mathrm{HC}}=\sigma+\delta$.

For two proteins that experience only their mutual interactions, the motion of the common center-of-friction completely decouples from their relative motion. Let us first look a this relative motion, which then corresponds to a driven diffusive motion of a point-particle around a sphere. The transition probability for the relative coordinate in the vicinity of the excluded hard-core region is shown in Fig. 8. The time step is chosen to be $\mathrm{d} t=0.001 \mathrm{~ns}$ so that $\mathcal{O}\left(\sqrt{\left\langle\vec{r}^{2}\right\rangle-\langle\vec{r}\rangle^{2}}\right) \sim 0.02 \mathrm{~nm}$. Therefore, we expect that the curvature of the excluded hard-core sphere does not lead to considerable systematic deviations from the exact transition probability. The numerically obtained transition probabilities for one integration step are compared with the 'exact' transition probability that has been calculated by solving the corresponding Fokker-Planck equation.

\section{Mean first passage time}

As an example of a derived quantity we look more closely at the mean first passage time for two different driven diffusive processes where hard-wall interactions play an important role (see also Fig. [6 for a first brief example). Although these cases capture typical situations one encounters in simulations of soft matter systems, they are simple enough so that the mean first passage time obtained from the numerical integration of the
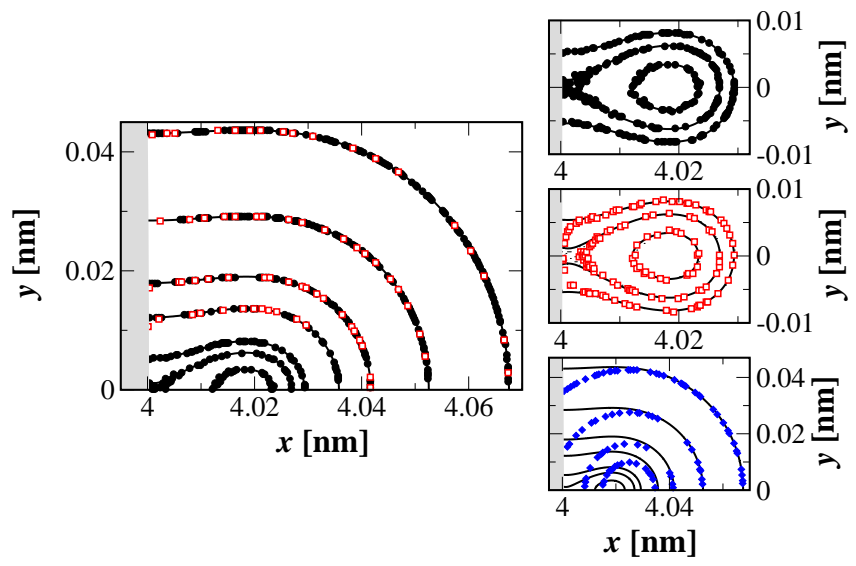

FIG. 8. Contour plot of the transition probability of the relative vector of two proteins. Its evolution corresponds to a point particle with $D=D_{1}+D_{2}=0.24 \mathrm{~nm}^{2} / \mathrm{ns}$. The relative coordinate is initially $\vec{r}(0)=(4.025 \mathrm{~nm}, 0 \mathrm{~nm})$ and evolves for the time $t=0.001 \mathrm{~ns}$. The force (for the relative component, compare relation (34)) is obtained from (42) with $K=4 k_{\mathrm{B}} T_{\text {room }} \mathrm{nm}, \kappa=0.02 \mathrm{~nm}^{-1}, \sigma=3.6 \mathrm{~nm}$, $A=3 k_{\mathrm{B}} T_{\text {room }}$ and $\delta=0.4 \mathrm{~nm}$ (compare e.g. Ref. 40) making up an excluded hard-core region of radius $R_{\mathrm{HC}}=4 \mathrm{~nm}$. The numerical results are obtained from one integration step (i.e. $\mathrm{d} t=0.001 \mathrm{~ns}$ ) for which the encounter frequency is approximately $12.7 \%$. Exact curves obtained by solving the Fokker-Planck equation are displayed by solid lines. Left panel: (black) filled circles for our new algorithm, open (red) squares for event-driven scheme, for symmetry reasons only positive $y$ are shown. For the inner contour levels results from our method are shown separately in the upper right panel, those from the event-driven are shown in the middle panel on the right. The lower right panel exhibits the same data for the rejection scheme (blue filled diamonds). The grey shading represents the region which is inaccessible to the relative coordinate.

equations of motion can be compared with an analytical solution. The latter is obtained from the mean escape time from an interval $[a, b]$ with a reflecting boundary at $a$ and an adsorbing boundary at $b$,

$$
\tau\left(q_{0}\right)=\frac{1}{D} \int_{q_{0}}^{b} \mathrm{~d} q \exp (\beta \Phi(q)) \int_{a}^{q} \mathrm{~d} q^{\prime} \exp \left(-\beta \Phi\left(q^{\prime}\right)\right)
$$

for a particle at inverse temperature $\beta=1 / k_{\mathrm{B}} T$ moving in a potential $\Phi(q)$, and starting at $q_{0} \underline{\underline{6}}$

In the first situation we continue the example from the previous section and consider the mean time the two proteins need to depart a certain distance $b$. This is related to a thermally activated process where the reaction coordinate is the separation $r=|\vec{r}|=\left|\vec{r}_{2}-\vec{r}_{1}\right|$. Taking the interaction potential (42) the problem is radially symmetric suggesting a transformation to polar coordinates. Due to the isotropy of the fluctuating forces in the Langevin equation the corresponding random forces in orthogonal polar coordinates are uncorrelated. There- 
fore, the escape time is only determined by the evolution equation for the separation $r$ which can be obtained from (34) by applying Itô's formula

$$
\mathrm{d} r=-\frac{1}{\eta_{r}} \frac{\mathrm{d}}{\mathrm{d} r} \Phi(r) \mathrm{d} t+\sqrt{2 D_{r}} \mathrm{~d} W_{r}
$$

where $\eta_{r}=k_{\mathrm{B}} T / D_{r}$ is the friction associated with the relative coordinate $\left(D_{r}=D_{1}+D_{2}\right)$ and $\mathrm{d} W_{r}$ is an incremental Wiener process. The effective potential is given by $\Phi(r)=\phi(r)-k_{\mathrm{B}} T \ln r$ in two space dimensions. Figure 9 depicts the exact values of the escape time as a function of the threshold $b$ together with the numerically obtained values. Before the particle crosses the threshold at $b$ many integration steps have to be carried out. In addition, a small time step $\mathrm{d} t$ has to be chosen to capture the variations in the force field so that only a small fraction of encounter events will occur. It is thus expected that the heuristic algorithms will produce good results. Figure 9 indeed confirms this expectation. This example points out that the rejection scheme may produce accurate results for certain observables. However, this does not necessarily mean that the rejection procedure is reliably applicable in general situations, as already demonstrated with the examples above, and as will be underlined by the following findings for the mean first passage time of a particle close to a wall.

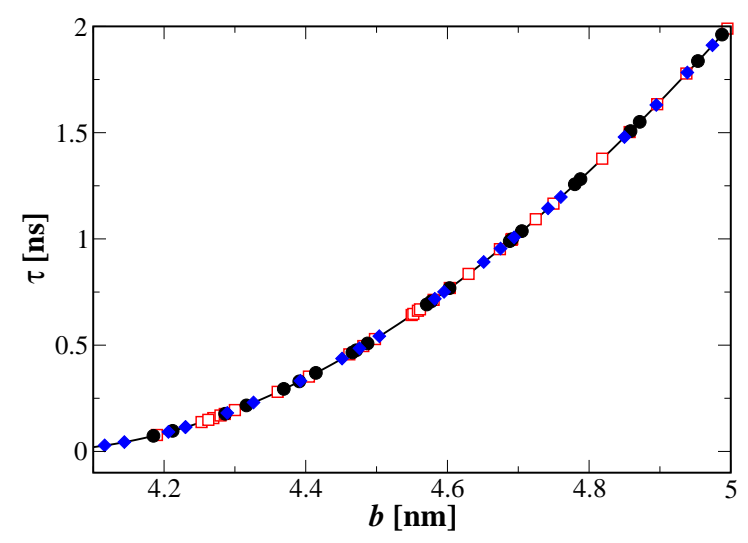

FIG. 9. Escape time $\tau$ from the potential (42) as a function of the location $b$ of the (radial) threshold. The same potential parameters as in Fig. 8 are used with $\vec{r}(0)=(4.025 \mathrm{~nm}, 0 \mathrm{~nm})$ and $\mathrm{d} t=0.001 \mathrm{~ns}$. With increasing $b$ the percentage of time steps that involve a 'collision' with the hard-core region drops from approximately $13 \%$ to $2 \%$ for the shown data. The numerical data is shown by (black) circles for our proposed method, by (red) open squares for the event-driven scheme and by (blue) diamonds for the rejection scheme, the solid curve represents the exact escape time evaluated from (43) with $a=R_{\mathrm{HC}}=4 \mathrm{~nm}, q_{0}=4.025 \mathrm{~nm}$ and $\Phi(q)=\phi(q)-k_{\mathrm{B}} T \ln r$ (see (42) and main text). The mean escape time was measured from the simulations as described in Refs. 9 and 36 .

As a second example we consider a (colloidal) particle which hits a hard wall (for instance, a wall or obstacle in a microfluidic device or an organelle in a cell etc.). The particle is driven by a deterministic force with a non-vanishing component perpendicular to the wall (for example due to an electric or magnetic field). For simplicity, we restrict ourselves again to two dimensions and assume that the wall located at $x=0$ is infinitely extended along the $y$ direction such that it confines the particle to $x>0$ and $y \in \mathbb{R}$. The particle is subject to the force $\vec{F}=(-f, f)$. In Fig. 10 we show the mean time a particle of radius $1 \mu \mathrm{m}$ starting at $\vec{r}(0)=(0,2 \mu \mathrm{m})$ needs to 'slide along the wall' by a distance of 10 particle radii until it crosses the line at $b=y=10 \mu \mathrm{m}$. The results obtained for the different integration schemes are compared to the exact solution calculated from (43) with $\Phi(q)=-f q$ to be $\tau=b \eta / f$. While our new integration scheme yields the exact passage times, the rejection scheme dramatically fails for too large $f$ as it artificially slows down the particle motion due to prevailing rejection steps until it practically stops completely. This goes in hand with a considerable increased computing time, see inset of Fig. 10. The event-driven scheme produces very good results. However, it requires (exponentially) increasing computation time in the case of high collision frequency and strong forces into the wall as many realizations of the random force for the propagation step away from the wall will produce invalid end positions.

\section{Computational efficiency}

To analyze the computational efficiency of our algorithm in comparison to the rejection and event driven scheme in some more detail and to get an impression of its performance in realistic systems, we come back to the above example of the two interacting proteins. As in realistic applications, we simulate the evolution of the individual coordinates of the two interacting particles, i.e. we do not decouple the center-of-friction motion. The event-driven scheme requires the determination of the collision time of the two spheres once an overlap has been detected. This collision time can be determined analytically for this special case by solving a quartic equation for non-vanishing forces (for zero force it reduces to a quadratic equation). Having the possible generalization of an arbitrarily shaped rigid particle in mind, however, we calculated the collision time by an iterative scheme which was used by Tao et al $\underline{\underline{18}}$ They determined the collision time by subdividing the integration step, which led to a particle overlap, into 100 sub-steps to successively propagate the particles along the "Euler path" until the collision is detected. Alternatively, the collision time can be determined by using nested intervals. Our method also requires the (numerical) evaluation of a root when determining the modified normal component of the relative motion (see Appendix $\mathrm{A}$ ). However, the motion perpendicular to the normal component and, in particular, the evolution of the center coordinate need not be altered and are indeed kept unchanged by the algorithm, so that 


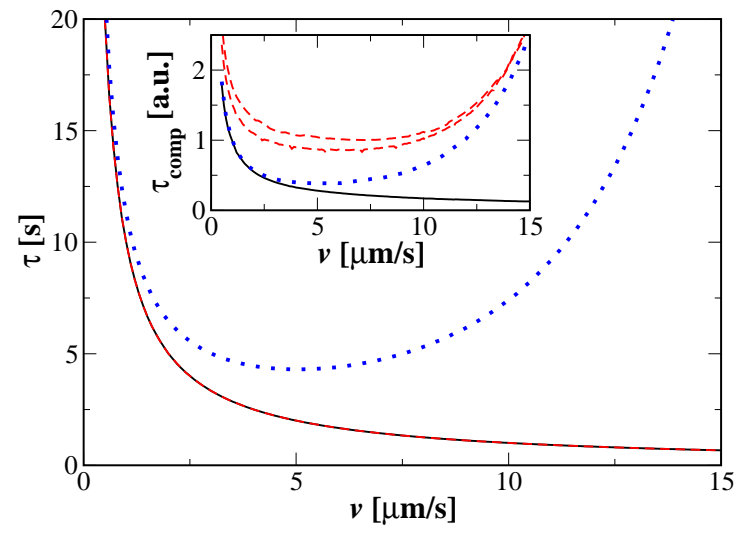

FIG. 10. Mean first passage time of a particle (radius $1 \mu \mathrm{m}$ ) close to a wall (located at $x=0$ ) as a function of $v=f / \eta$. It moves from $\vec{r}(0)=(0,2 \mu \mathrm{m})$ to the line $y=b=10 \mu \mathrm{m}$ driven by the force $\vec{F}=(-f, f)$ (see main text). (For fixed $f$ the convergence as a function of $\mathrm{d} t$ is similar to Fig. 6] not shown.) The numerical data is shown by the (black) line for our method, which is indistinguishable from the exact passage time $\tau=b / v$ evaluated from (43) with $a=-\infty, b=10 \mu \mathrm{m}$, $q_{0}=0$ and $\Phi(q)=-f q=-\eta v q$. The (red) dashed line represents the event-driven scheme and the (blue) dotted line the rejection scheme (time step $\mathrm{d} t=0.01 \mathrm{~s}$ in all cases). The mean escape time was measured from the simulations as described in Refs. 9 and 36. The inset shows the computation time consumed by the different algorithms. (See Sec. VID on how the collision time is determined for the event-driven scheme. Upper dashed curve: stepwise propagation; lower dashed curve: nested intervals.) For larger driving force, the computation times of the rejection and event-driven scheme is an order of magnitude longer as compared to our algorithm. For the shown data the 'collision' frequency increases from a few per cent to around $90 \%$.

only a one-dimensional motion is affected. We therefore expect our algorithm to be faster than the event-driven scheme where all components are simultaneously affected when accounting for hard bodies. For the typical parameters of the exemplary protein system from above, see for example Fig. 8, we indeed found that the required computing time of our method is smaller than the time for the event-driven scheme. Not surprisingly, the discrepancy increases for increasing encounter frequencies $w$. For the data presented in this work we typically observe a difference of $10 \%$ to $30 \%$ in computational speed. The rejection scheme, on the other hand, is typically $20 \%$ faster at identical time steps. However, to diminish the systematic errors due to encounter events with hard walls the time step has to be reduced accordingly by at least one magnitude, thus requiring considerably increased computation time.

For a more quantitative analysis we investigate the necessary computational efforts to achieve a prescribed accuracy. To this end we again use the passage time problem of a colloid gliding along a wall of the previous subsection as for this example analytical results can be
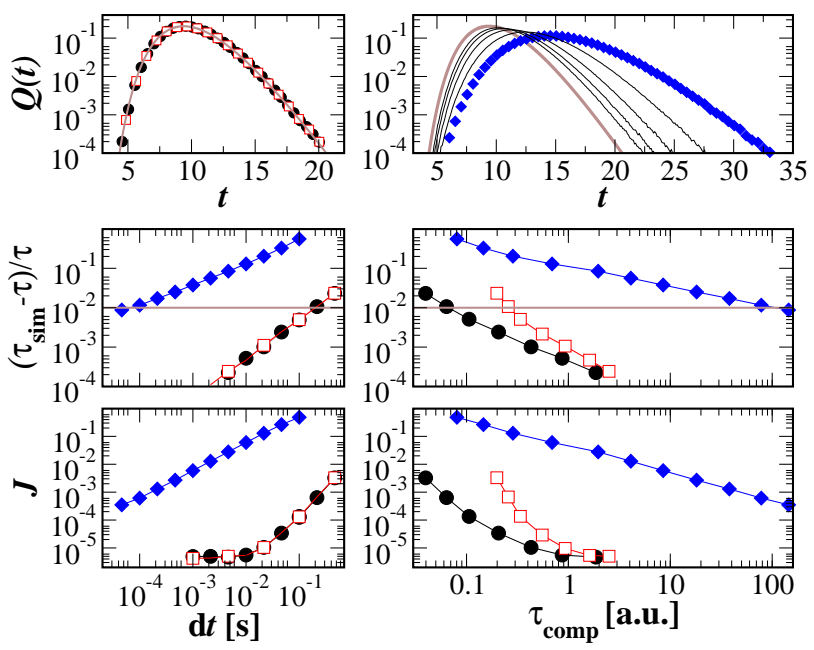

FIG. 11. First passage time distribution for the system described in Fig. [10 with $v=1 \mu \mathrm{m} / \mathrm{s}$. Upper left panel: logarithm of the simulated distribution of our method (black circles) and of the event driven scheme (open red squares) together with the exact curve (solid brown line) for $\mathrm{d} t=0.1 \mathrm{~s}$; upper right panel: same comparison for the rejection scheme (blue diamonds) and in addition curves for smaller $\mathrm{d} t$ (thin black lines, $\left.\mathrm{d} t=10^{-4 / 3}, 10^{-5 / 3}, 10^{-2}, 10^{-7 / 3} \mathrm{~s}\right)$. The middle panels show the relative deviation of the simulated mean first passage time $\tau_{\text {sim }}$ from the exact value $\tau=10 \mathrm{~s}$, the lower panels show the Jensen-Shannon divergence (46). Both quantities are shown in double-logarithmic depiction as a function of the time step (left hand side) and the computation time (right hand side, the number of realizations is fixed to $10^{7}$, $\mathrm{d} t$ is varied, nested intervals are used for the event-driven method). Note the different order of magnitude for the different methods (our method: black circles; event driven: open red squares; rejection: blue diamonds). The horizontal brown line indicates a prescribed accuracy of $\left(\tau_{\text {sim }}-\tau\right) / \tau=1 \%$. For this setting the event driven-scheme exhibits a rather large encounter frequency of about $50 \%$ with the wall and needs by a factor three more computation time than our methods. For $\mathrm{d} t=0.01 \mathrm{~s}$ (compare Fig. 10) an accuracy of $\left(\tau_{\text {sim }}-\tau\right) / \tau \approx 0.5 \%$ is achieved, the event-driven scheme then produces an encounter frequency of $11 \%$ and needs roughly $55 \%$ more computation time. In contrast, the rejection scheme shows a very slow 'convergence'.

obtained. For the system described in Fig. 10 (starting point $y_{0}=0$ ) the first passage time distribution $Q(t)$ can be calculated $\underline{41}$ to be

$$
Q(t)=\frac{|b|}{\sqrt{4 \pi D t^{3}}} \exp \left(-\frac{(b-v t)^{2}}{4 D t}\right) .
$$

In addition to analyzing the relative deviation of the simulated mean first passage time $\tau_{\text {sim }}$ from the analytical result $\tau=b \eta / f$ we will compare this exact distribution $Q$ with the simulated curve $Q_{\text {sim }}$ by determining the JensenShannon divergence $\underline{\underline{42}}$ as a measure for the distance between $Q$ and $Q_{\text {sim }}$. The Jensen-Shannon divergence is 
defined by

$$
J=\frac{1}{2 \ln 2} \int_{0}^{\infty} \mathrm{d} t\left(Q(t) \ln \frac{Q(t)}{M(t)}+Q_{\operatorname{sim}}(t) \ln \frac{Q_{\operatorname{sim}}(t)}{M(t)}\right)
$$

where $M=\left(Q+Q_{\text {sim }}\right) / 2 . J$ can take on values between 0 and 1 with 0 indicating identical distributions $\underline{43}$ The findings for the system of Fig. [10 are shown in Fig. [1]. Surprisingly the event-driven scheme produces results of the same accuracy as our new methods for the same time step. Therefore, the computational difference is solely determined by the effort for a single integration step which amounts to typically 10-30 \% for moderate encounter frequencies with the wall smaller than $10 \%$. In accordance with our general observation the data in Fig. 11 point out again that a rather small time step has to be chosen for the rejection scheme to match the accuracy of the other methods.

\section{CONCLUSIONS AND OUTLOOK}

The explicit form of the transition probability for a Brownian particle in a constant force field on the onedimensional half-line shows that multiple encounters with hard bodies may lead to contributions to that transition probability that cannot be generated by a direct numerical integration of the Langevin equations. This results in unsystematic and uncontrolled errors in heuristic integration schemes, even for very small time steps. For an algorithm whose accuracy is to be systematically controlled by the size of the discretization time step, the recourse to the transition probability seems unavoidable.

In this article we have developed a novel algorithm to account for hard-body interactions in Brownian dynamics simulations with controllable discretization error, and compared its performance with heuristic schemes from the existing literature. The central idea is that, once a hard-body interaction has been detected during the numerical integration of the Langevin equations of motion, the component of the particle displacement(s) involved in the 'collision' is generated directly from the solution for the transition probability in the presence of a hard reflecting wall. We demonstrated that the aforementioned transition probability for the one-dimensional half-line can be used to construct an approximate solution for general many-dimensional physical systems with hardbody interactions. The algorithm decomposes the particle motion into parts that are unaffected by the hardbody interaction and into an 'affected' part along which the hard-wall 'collision' occurs. The latter corresponds to the relative motion of the collision partners and is reduced a one-dimensional motion on a half-line with a hard, reflecting boundary at the origin (the point of 'collision'). For this setting, the general time-dependent transition probability is known analytically, and can be used to generate relative displacements which are consistent with the hard-body interactions and with the statistical properties of the underlying stochastic evolution.

This principle can be extended to more general situations, for example, where structured particles with a nonspherical shape are present. In general, the decomposition of the full particle displacement(s) has to be adopted in such a way that the components 'unaffected' by the hard-body interaction and the 'affected' component statistically decouple from each other (for the considered time step), so that the hard-body 'collision' can be corrected without interfering with the rest of the particle motions. The 'unaffected' displacement is thus retained (to save computation time) and together with the corrected 'collision' part is used to reconstruct the overall displacements of the involved particles. In this work we developed this procedure for the case of interacting spherical particles, the generalization to more complicatedly structured rigid particles will be presented in a separate article. Furthermore, situations where multiple particle overlaps occur, for example in dense systems, and where secondary overlaps due to the applied modifications show up will also be treated elsewhere.

\section{ACKNOWLEDGMENTS}

Financial support of the SFB 613 and the SFB 625 is gratefully acknowledged. We thank the experimental biophysics group of the Bielefeld University for providing computational resources.

\section{Appendix A: Generation of random numers distributed according to (8)}

One of the central building blocks of the described algorithm is the generation of a random number $q$ that is distributed according to (8). This can be achieved by using a general transformation method (see e.g. 9 or 44). Consider a distribution function $p$ and define $F(q)=\int_{0}^{q} \mathrm{~d} q^{\prime} p\left(q^{\prime}\right)$. If the number $x$ is uniformly distributed on $[0,1]$ then $q=F^{-1}(x)$ is distributed according to the distribution $p$. Here $F^{-1}$ is the inverse of $F$.

For the distribution function $p(q)=p_{\mathrm{H}}\left(q, q_{0}, \mathrm{~d} t, v, D_{q}\right)$ with $p_{\mathrm{H}}$ given in (8) the corresponding integral is

$$
F(q)=\frac{\operatorname{erfc}\left(\frac{q_{0}+v \mathrm{~d} t}{\sqrt{4 D_{q} \mathrm{~d} t}}\right)-\exp \left(\frac{v q}{D_{q}}\right) \operatorname{erfc}\left(\frac{q+q_{0}+v \mathrm{~d} t}{\sqrt{4 D_{q} \mathrm{~d} t}}\right)}{\operatorname{erfc}\left(\frac{q_{0}+v \mathrm{~d} t}{\sqrt{4 D_{q} \mathrm{~d} t}}\right)}
$$

Due to the monotony of $F$ the equation $x=F(q)$ has precisely one solution $q$ for given $x$. As to the best of our knowledge there is no closed expression for the inverse of $F, q$ is obtained by solving $x=F(q)$ numerically using, for example, Newton's methods, bisection methods or combinations thereof. In this work Brent's scheme $\underline{\underline{44}}$ was used from the GNU scientific library $\underline{45}$. 


\section{Appendix B: Harmonic oscillator on the half-line}

Let us consider a Brownian particle on a half-line in a harmonic potential with spring constant $k$, i. e. the total potential is given by

$$
\phi(q)= \begin{cases}\frac{1}{2} k q^{2}, & \text { if } q \geq 0 \\ \infty, & \text { if } q<0\end{cases}
$$

with $k>0$. Introducing the characteristic time $\tau=\eta / k$ the corresponding overdamped Langevin equation reads

$$
\mathrm{d} q=-\frac{q}{\tau} \mathrm{d} t+\sqrt{2 D_{q}} \mathrm{~d} W
$$

with a reflective boundary at $q=0$. For the unrestricted harmonic potential on the real axis the transition probability $p\left(q, t ; q_{0}\right)$ of the Ornstein-Uhlenbeck process (B2) is analytically known ${ }^{7.8}$ for initial condition $p\left(q, t=0 ; q_{0}\right)=\delta\left(q-q_{0}\right)$ :

$$
p_{\mathrm{OU}}\left(q, t ; q_{0}\right)=\frac{1}{\sqrt{2 \pi \tau D_{q}\left(1-s^{2}\right)}} \exp \left(-\frac{\left(q-s q_{0}\right)^{2}}{2 \tau D_{q}\left(1-s^{2}\right)}\right)
$$

where

$$
s=s(t)=\exp \left(-\frac{t}{\tau}\right)
$$

This solution can be used to construct the transition probability for the harmonic oscillator (B1) and reflecting boundary condition on $\mathbb{R}_{>0}$ by using the image method:

$$
p\left(q, t ; q_{0}\right)=p_{\mathrm{OU}}\left(q, t ; q_{0}\right)+p_{\mathrm{OU}}\left(-q, t ; q_{0}\right) .
$$

Figure 12 compares this exact solution with the numerically obtained transition probability using the algorithms discussed in the main text. To account for the variations in the force field the integration time step is chosen to be $\mathrm{d} t=0.01 \tau$. This time step is already small enough that the solution from the event driven scheme is of similar numerical accuracy as our new integration scheme. For the rejection method, however, there are still deviations close to the wall due to the insufficient treatment of the reflecting boundary condition.

The transition probability of the unrestricted Ornstein-Uhlenbeck process on $\mathbb{R}$ is a Gaussian. Therefore, an exact integration algorithm for any $\mathrm{d} t$ exists 46 :

$$
q(t+\mathrm{d} t)=q(t) s(\mathrm{~d} t)+\sqrt{\tau D_{q}\left(1-[s(\mathrm{~d} t)]^{2}\right)} G
$$

with $G$ being a random Gaussian number of mean zero and variance one and $s(\mathrm{~d} t)$ given in (B4). Due to the symmetric structure of the transition probability (B5) one can readily construct an exact update scheme to generate valid positions $q^{*}$ for the potential (B1) with a hard wall at the center as well:

1. Calculate a 'preliminary' position $q(t+\mathrm{d} t)$ according to (B6) by a standard integration algorithm.
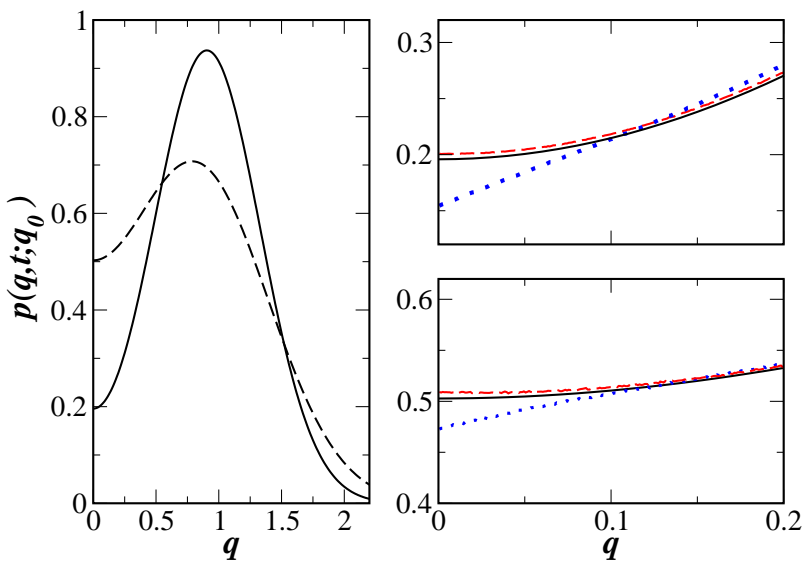

FIG. 12. Transition probability of a particle on a half-line in the harmonic potential (B1) for the parameters $\tau=1.0$, $D_{q}=1.0$ and initial position $q_{0}=1.0$. In the left panel the analytical solution (compare (B5) and (B3) ) is displayed for two times, namely $t=0.1$ (solid line) and $t=0.2$ (dashed line).$^{28}$ For the generation of the numerical data the rejection method and our scheme is used with a time step $\mathrm{d} t=0.01 \tau=0.01$. (The results from the event-driven algorithm are not displayed as they are indistinguishable from our method). The upper right panel shows a blow up for $t=0.1$, the lower one for $t=0.2$ (same magnification factor). For our new method (red dashed lines) there are still tiny deviations which are related to the finite discretization size of the time step. The rejection method (blue dotted lines) suffers from errors close to the wall due to the insufficient representation of hard-wall interactions.

2 a. If this proposed $q$ is in the physical domain, i.e. $q>0$, it is accepted as the new position, $q^{*}=q$.

$2 \mathrm{~b}$. If this proposed position is unphysical, i.e. $q<0$, a new physical position $q^{*}>0$ is obtained by simply reflecting $q$, i.e. $q^{*}=-q$.

We finally remark that this procedure, the construction of valid end positions by reflection, is possible because the exact solution can be obtained by the image method. For $k \rightarrow 0$ the system reduces to free diffusion on a half-line and the described method reduces to the one discussed in Ref. 17 in the special case of one space dimension.

${ }^{1}$ R. A. L. Jones, Soft Condensed Matter (OUP, Oxford 2002).

${ }^{2}$ J. K. G. Dhont, An Introduction to Dynamics of Colloids (Elsevier, Amsterdam, 2003).

${ }^{3}$ H. C. Öttinger, Stochastic Processes in Polymeric Fluids (Springer, Berlin 1996).

${ }^{4}$ M. Doi and S. F. Edwards, The Theory of Polymer Dynamics (Clarendon Press, Oxford, 1986).

${ }^{5}$ M. B. Jackson, Molecular and Cellular Biophysics (CUP, Cambridge, 2006).

${ }^{6}$ C. W. Gardiner, Handbook of Stochastic Methods (Springer, Berlin, 1983).

${ }^{7}$ H. Risken, The Fokker-Planck Equation (Springer, Berlin, 1984). ${ }^{8}$ N. G. van Kampen, Stochastic Processes in Phsyics and Chemistry (North Holland, Amsterdam, 1987).

${ }^{9}$ J. Honerkamp, Stochastische Dynamische Systeme (VHC, Weinheim, 1990; english translation: Stochastic Dynamical Systems, Wiley-VHC, 1993). 
${ }^{10}$ N.-T. Nguyen and S. T. Wereley, Fundamentals and Applications of Microfluidic, (Artech House, 2006).

${ }^{11}$ P. E. Kloeden and E. Platen, Numerical Solution of Stochastic Differential Equations, 3rd ed. (Springer, Berlin, 1999).

${ }^{12}$ I. Snook, The Langevin and Generalised Langevin Approach to the Dynamics of Atomic, Polymeric and Colloidal Systems (Elsevier, Amsterdam, 2007).

${ }^{13}$ For walls with absorbing boundary conditions similar problems arise, see e.g. Th. M. A. O. M. Barenbrug, E. A. J. F. Peters, and J. D. Schieber, J. Chem. Phys. 117, 9202 (2002) or E. A. J. F. Peters and Th. M. A. O. M. Barenbrug, Phys. Rev. E 66 056701 (2002).

${ }^{14}$ B. Cichocki and K. Hinsen, Physica A 166, 473 (1990).

${ }^{15}$ W. Schaertl and H. Sillescu, J. Stat. Phys. 74, 687 (1994).

${ }^{16}$ P. Strating, Phys. Rev. E 59, 2175 (1999).

${ }^{17}$ A. Scala. Th. Voigtmann, and C. De Michele, J. Chem. Phys. 126, 134109 (2007).

${ }^{18}$ Y.-G. Tao, W. K. den Otter, J. K. G. Dhont, and W. J. Briels, J. Chem. Phys. 124, 134906 (2006).

${ }^{19}$ The scheme of Scala et al 17 can be developed systematically and envolves approximations which can be controlled within the numerics. However, this method works only for systems with vanishing (deterministic) forces.

${ }^{20} \mathrm{H}$. Behringer and R. Eichhorn, Phys. Rev. E 83, 065701(R) (2011).

${ }^{21} \mathrm{G}$. Roepstorff, Pfadintegrale in der Quantenphysik (Vieweg, Braunschweig, 1991; english translation: Path Integral Approach to Quantum Physics, Springer, 1996).

${ }^{22} \mathrm{~W}$. Paul and J. Baschnagel, Stochastic Porcesses (Springer, Berlin 1999).

${ }^{23}$ A. Greiner, W. Strittmatter, and J. Honerkamp, J. Stat. Phys. 51, 95 (1988).

${ }^{24}$ Details of heuristic methods proposed in the literature ${ }^{14}-18$ will be further discussed in Sec. III below for the concrete example of a Brownian particle on an infinite half-line with a hard wall at the origin.

${ }^{25}$ M. V. Smoluchowski. Phys. Z. 17, 557 (1916)

${ }^{26} \mathrm{~S}$. Chandrasekhar, Rev. Mod. Phys. 15, 1 (1943).

${ }^{27}$ M. Schmidt, C. P. Royall, A. van Blaaderen, and J. Dzubiella, J. Phys.: Condens. Matter 20, 494222 (2008).

${ }^{28} \mathrm{As}$ we do not have a particular system or physical context in mind, all parameters in Figs. 1 2 13 and 4) (as well as 12) are given in terms of characteristic times, lengths, diffusion constants etc.

${ }^{29}$ The heuristic scheme of Ref. 15 removes an unphysical overlap of two hard spheres by displacing them along their connecting line until the surfaces are in contact. This evidently produces the same single-step distribution as the rejection scheme with the difference that the delta-like peak is moved to the wall (i. e. $q=0$ in Fig. 1).

${ }^{30}$ G. Lamm and K. Schulten, J. Chem. Phys. 75, 365 (1981).

${ }^{31}$ R. Friedrich et al., Phys. Lett. A 271, 217 (2000).

${ }^{32}$ R. Friedrich, J. Peinke, M. Sahimi, and M. Reza Rahimi Tabar, Phys. Rep. 506, 87 (2011).

${ }^{33}$ Strictly speaking, we also have to assume that other hard boundaries or other particles, which may be present in the system, are sufficiently far away to not influence the considered displacement step (i. e. at least several standard deviations of the typical displacement). Obviously, this cannot always be guaranteed, for example, at corners of a structured boundary or for very dense suspensions. An extension of our algorithm to include the case of 'multiple collisions' is subject of current research.

${ }^{34} \mathrm{D}$. Leckband and J. Israelachvili, Q. Rev. Biophys. 34, 105 (2001).

${ }^{35}$ G. Schreiber, G. Haran, and H.-X. Zhou, Chem. Rev, 109, 839 (2009).

${ }^{36}$ R. Mannella, Phys. Lett. A 254, 257 (1999).

${ }^{37}$ I. Proudman and J. R. A. Pearson, J. Fluid Mech. 2, 237 (1957).

${ }^{38}$ D. J. Acheson, Elementary Fluid Dynamics (OUP, Oxford, 1990).

${ }^{39}$ A. Tardieu et al., J. Cryst. Growth 196, 193 (1999).

${ }^{40}$ G. Pellicane, D. Costa and C. Caccamo, J. Phys. Condens. Matter 16, S4923 (2004).

${ }^{41}$ I. Karatzas and S. E. Shreve, Brownian motion and stochastic calculus (Springer Verlag, New York, Berlin, 1988).

${ }^{42}$ J. Lin, IEEE Trans. Inform. Theory 37, 145 (1991).

${ }^{43}$ For the normal distribution $\mathcal{N}\left(\mu, \sigma^{2}\right)$ the two distributions $\mathcal{N}(0,1)$ and $\mathcal{N}(0.1,1)$ have a Jenson-Shannon divergence 0.00125 , the distributions $\mathcal{N}(0,1)$ and $\mathcal{N}(0.2,1)$ a divergence 0.005 .

${ }^{44}$ W. H. Press, S. A. Teukolsky, W. T. Vetterling, and B. P. Flannery, Numerical Recipies, 3rd ed. (CUP, Cambridge, 2007)

${ }^{45} \mathrm{M}$. Galassi et al., GNU Scientific Library Reference Manual, 3rd ed. (Network Theory Limited., 2009), http://www.gnu.org/software/gsl/.

${ }^{46}$ D. T. Gillespie, Phys. Rev. E 54, 2084 (1996). 